\title{
Constructive Modalities with Provability Smack
}

\section{Author's Cut, v. 2.04: updated and extended electronic version}

\author{
Tadeusz Litak \\ The references were last updated in 2015
}

To the memory of Leo Esakia and Dito Pataraia

\begin{abstract}
I overview the work of the Tbilisi school on intuitionistic modal logics of wellfounded/scattered structures and its connections with contemporary theoretical computer science. Fixed-point theorems and their consequences are of particular interest.
\end{abstract}

\section{Contents}

1 Introduction

2 A Primer on Intuitionistic Modalities 3

2.1 Relational Semantics . . . . . . . . . . . . . . . 6

3 The Fixpoint Theorem 9

4 Leo Esakia and Extensions of $\mathrm{mHC}$

$4.1 \mathrm{mHC}$ and Topological Derivative ............. 13

$4.2 \mathrm{KM}$ and Scatteredness . . . . . . . . . . . . . . 15

4.3 Completeness, Lattice Isomorphisms and Bimodal Translations . . 16

5 Scattered Toposes $\quad 19$

5.1 Preliminaries on Topos Logic . . . . . . . . . . . . . . . 19

5.2 Non-expansive Morphisms, Fixpoints and Scattered Toposes . . . . 24

5.3 Topos of Trees and Its Generalizations . . . . . . . . . . . 27

References 29

Disclaimer The paper is a modified version of Lit14, which has been commissioned for the highly recommended volume Bez14. Please make clear which version you are quoting, as even the numeration of all environments is very different than in the version printed in the volume (this is by no means the only difference). 


\section{Introduction}

The intended audience of [Bez14] is probably aware that much of Leo Esakia's research concentrated on semantics for the intuitionistic logic IPC, the modal logic $\mathrm{GL}^{\mathrm{cl}}$ of Löb, its weakening $w_{G r z}{ }^{c l}$ and intuitionistic-modal systems like the logic KM or its weakening $\mathrm{mHC}$; see Table 2 for all definitions. $\mathrm{GL}^{\mathrm{cl}}$ is also known as the Gödel-Löb logic, but this name may suggest more personal involvement with the system than Gödel ever had; $\mathrm{KM}$ or $\mathrm{mHC}$ will be discussed in Section 4. A central feature of semantics for such systems is well-foundedness or scatteredness. While in the case of IPC well-foundedness is a sufficient, but not necessary condition - intuitionistic logic is complete wrt well-founded or even finite partial orders, but sound wrt much bigger class of structures $-\mathrm{GL}^{\mathrm{cl}}$ and $\mathrm{KM}$ require it even for soundness. This is due to the fact that the latter two systems include a form of an explicit induction axiom: in the case of $\mathrm{GL}^{\mathrm{cl}}$ the well-known Löb axiom (which here will be called the weak Löb axiom) and in the case of KM the strong Löb axiom - less well-known to modal logicians, but as we are going to see, better known to type theorists. Scatteredness is the topological generalization of well-foundedness; Simmons [Sim82] provided tools necessary to define its point-free counterpart and the Tbilisi school noted that this notion also makes sense in the topos setting. In fact, the most generic way of defining scateredness is via modal syntax: as validity of the Löb principle for a suitable "later" modality.

The interplay of relational, topological, point-free and algebraic aspects in the above paragraph should not feel unnatural to anybody familiar with Leo's attitude to research. Let us look at an important example how results can travel from one setting to another. In the mid-1970's, it was established that Löb-like logics enjoy the so-called Fixpoint Theorem. At first, the intention was to grasp the algebraic content of Gödel's Diagonalization Lemma. Yet in its own right it turned out to be one of the most fascinating results ever proved about such systems. Section 3 gives an overview of some of its applications and consequences. For now, let us just mention that Leo Esakia used it, e.g., to characterize algebras for KM, see Theorem 14 and Corollary 15 here. Furthermore, it seems to have inspired the work on scattered toposes: [EJP00, Section 3] claims to present its topos-theoretic counterpart. However, as the result central for the topos version (Theorem 40 here) does not even include modalities in its formulation, the word counterpart has to be understood rather loosely.

As we will see, in hindsight [EJP00] turns out to be closely connected to very recent developments in Theoretical Computer Science, in particular the work of Birkedal et al. on the topos of trees [BMSS12], itself an example of a scattered topos. Thus, it seems particularly regrettable that the spadework of the Tbilisi school has not been carried further and is not more widely known.

The paper is structured as follows. Section 2 recalls syntactic and semantic basics of intuitionistic normal modal logics. Section 3 focuses on fixpoint results for Löb-like systems. Section 4 introduces the work of the Georgian school on extensions of mHC. Finally, Section 5 discusses scattered toposes, beginning with an overview of the topos logic.

While the work is intended as an overview and claims to novelty are minimal, they are perhaps not entirely non-existent. Theorem 14 is the most general form of Esa06, Proposition 3] I could think of and Section 4.3 reproves results on extensions of $\mathrm{mHC}$ using the framework of WZ97; WZ98; in fact, it seems that Corollary 31 is the first published proof of the corresponding extension of the Blok-Esakia Theorem announced in Esa06. 
Table 1: Axioms for $\mathrm{K}^{\mathrm{i}}$

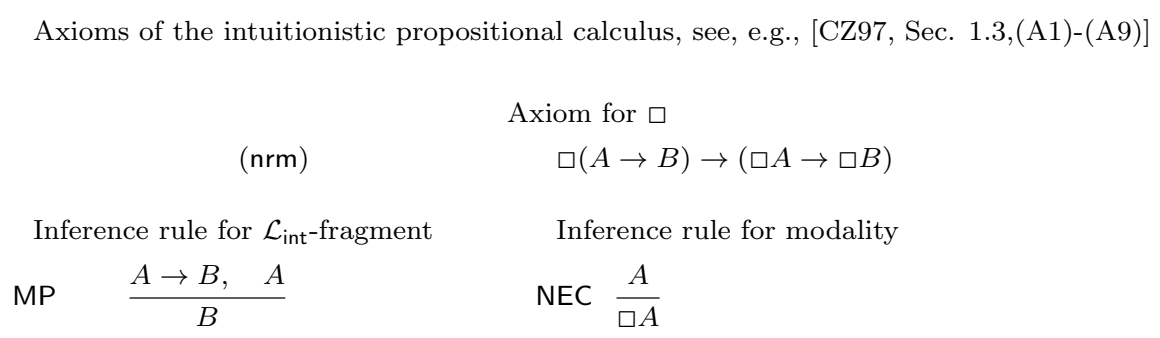

Remark 1. As a part of a larger project, I formalized most of syntactic derivations in the paper - in particular those relevant for Section 5.2 - in the Coq proof assistant. Readers interested in this ongoing project are welcome to contact me. The formalization covers, in particular, most of material in [Esa98], which prepared the proof-theoretical background for [EJP00] and inspired the title of this paper.

\section{A Primer on Intuitionistic Modalities}

Modal formulas over a supply of propositional variables $\Sigma$ are defined by

$$
A, B::=\perp|p| A \rightarrow B|A \wedge B| A \vee B \mid \square A
$$

where $p \in \Sigma$. The set is denoted by $\mathcal{L}_{\square \text { int }} \Sigma$, but unless explicitly stated otherwise, I will keep $\Sigma$ fixed throughout and drop it from the notation. The purely intuitionistic language (i.e., without $\square$ ) will be denoted by $\mathcal{L}_{\text {int }}$. Note that the syntax extended with a $\diamond$ operator, intuitionistically not definable from $\square$, is of no interest for us here.

$\Gamma \subseteq \mathcal{L}_{\text {Dint }}$ is a normal $\mathcal{L}_{\square \text { int }}$-logic or an intuitionistic normal modal logic if it is closed under rules and axioms from Table 1 plus the rule of substitution. For any $\Gamma, \Delta \subseteq \mathcal{L}_{\square \text { int }}, \Gamma \oplus \Delta$ will denote the closure of $\Gamma \cup \Delta$ under substitution and the rules MP and NEC. In the case of $\Delta=\{\alpha\}$, I will also write $\Gamma \oplus \alpha$. Occasionally, I will write $\Gamma+\Delta$ for the closure under substitution and MP, but without NEC. This notation is analogous to the one used in [CZ97.

$\mathrm{K}^{\mathrm{i}}$ is the smallest intuitionistic normal modal logic, i.e., IPC $\oplus(\mathrm{nrm})$. IPC - the intuitionistic propositional calculus - can be thus defined as the intersection of $\mathrm{K}^{\mathrm{i}}$ and $\mathcal{L}_{\text {int }}$. Table 2 provides a list of additional axioms and logics which will be of interest to us. $\mathrm{GL}^{\mathrm{i}}, \mathrm{SL}^{\mathrm{i}}, \mathrm{mHC}$ and $\mathrm{KM}$ are of particular importance. As we see in Table 2 and Figure 1 , there are several ways in which these and related systems can be axiomatized. In particular, we have

Theorem 2 (Ursini Urs79], following Smorynski for the classical case). The following formalisms have the same set of theorems:

1. $\mathrm{GL}^{\mathrm{i}}$ as defined in Table 2

2. $\mathrm{K} 4^{\mathrm{i}} \oplus \frac{\square A \rightarrow A}{A}$

3. $\mathrm{K}^{\mathrm{i}} \oplus \frac{\square A \rightarrow A}{\square A}$ 
Table 2: $\mathcal{L}_{\square \text { int }}$ axioms and logics. See, e.g., Sot84, WZ97; WZ98, for more (also in the syntax extended with a $\diamond$ operator). $\square A$ below abbreviates $A \wedge \square A$
$(\mathrm{cl}) \quad((B \rightarrow A) \rightarrow B) \rightarrow B$
$\mathrm{Cl}=\mathrm{IPC}+(\mathrm{cl})=\mathrm{IPC}+(\mathrm{em})$
(nrm) $\quad \square(A \rightarrow B) \rightarrow(\square A \rightarrow \square B)$
$\mathrm{K}^{\mathrm{i}}=\mathrm{IPC} \oplus(\mathrm{nrm})=\mathrm{IPC} \oplus(\mathrm{opr})$
(em) $\quad A \vee \neg A$
(opr) $\quad \square(A \wedge B) \leftrightarrow(\square A \wedge \square B)$
(trns) $\quad \square A \rightarrow \square \square A$
$\mathrm{K} 4^{\mathrm{i}}=\mathrm{K}^{\mathrm{i}} \oplus($ trns $)$
$\mathrm{K}^{\mathrm{cl}}=\mathrm{K}^{\mathrm{i}} \oplus \mathrm{Cl}$
(bind) $\quad \square \square A \rightarrow \square A$
$\mathrm{K}^{\mathrm{cl}}=\mathrm{K} 4^{\mathrm{i}} \oplus \mathrm{Cl}$
(r) $\quad A \rightarrow \square A$
$\mathrm{R}^{\mathrm{i}}=\mathrm{K}^{\mathrm{i}} \oplus(\mathrm{r})=\mathrm{K}^{\mathrm{i}} \oplus(\mathrm{fmap})$
$\mathrm{C} 4^{\mathrm{i}}=\mathrm{K}^{\mathrm{i}} \oplus$ (bind)
In using the symbol $\mathrm{R}$, I follow
(fmap) $\quad(A \rightarrow B) \rightarrow(\square A \rightarrow \square B)$
$\mathrm{T}^{\mathrm{i}}=\mathrm{K}^{\mathrm{i}} \oplus($ refl)
$\mathrm{S} 4^{\mathrm{i}}=\mathrm{T}^{\mathrm{i}} \oplus \mathrm{K} 4^{\mathrm{i}}$
$\operatorname{Triv}^{i}=\mathrm{T}^{\mathrm{i}}+\mathrm{R}^{\mathrm{i}}$
(refl) $\square A \rightarrow A$
Note that above $\mathrm{R}^{\mathrm{i}}, \oplus$ is the same as + FM97.
(pll) $\quad(A \vee \square \square A) \rightarrow \square A$

$$
\mathrm{PLL}^{\mathrm{i}}=\mathrm{K}^{\mathrm{i}} \oplus(\mathrm{pll})=\mathrm{C}^{\mathrm{i}} \oplus \mathrm{R}^{\mathrm{i}}
$$
(ufp) $\quad \square(B \leftrightarrow A[B / p]) \rightarrow(\odot(C \leftrightarrow A[C / p]) \rightarrow(B \leftrightarrow C))$
$\mathrm{GL}^{\mathrm{i}}=\mathrm{K}^{\mathrm{i}} \oplus($ wlöb $)=\mathrm{K} 4^{\mathrm{i}} \oplus$ (henk) $=\mathrm{K}^{\mathrm{i}} \oplus(\mathrm{ufp})$ (see Theorem 2 below)
$\mathrm{GL}^{\mathrm{cl}}=\mathrm{GL}^{\mathrm{i}} \oplus \mathrm{Cl}$
(wlöb) $\quad \square(\square A \rightarrow A) \rightarrow \square A \quad$ (henk) $\quad \square(A \leftrightarrow \square A) \rightarrow A$
(slöb) $\quad(\square A \rightarrow A) \rightarrow A$
$\mathrm{SL}^{\mathrm{i}}=\mathrm{K}^{\mathrm{i}} \oplus(\mathrm{s} l o ̈ b)=\mathrm{K}^{\mathrm{i}} \oplus(\mathrm{glb})=\mathrm{GL}^{\mathrm{i}}+\mathrm{R}^{\mathrm{i}}$
The form (glb) comes from Goldblatt Gol81.
(grz) $\quad \square(\square(A \rightarrow \square A) \rightarrow A) \rightarrow \square A$
$\mathrm{wGrz}=\mathrm{K} 4^{\mathrm{cl}} \oplus(\mathrm{grz})$
$(\mathrm{sgrz})$
$(\square A \rightarrow A) \rightarrow \square A$
Note we only consider here classical variants of (grz)
(next) $\quad \square A \rightarrow(((B \rightarrow A) \rightarrow B) \rightarrow B) \quad$ (derv) $\square A \rightarrow((B \rightarrow A) \vee B)$
$\mathrm{CB}^{\mathrm{i}}=\mathrm{K}^{\mathrm{i}} \oplus($ next $)=\mathrm{K}^{\mathrm{i}} \oplus($ derv $)$
$\mathrm{CB}^{\mathrm{i}}$ stands for Cantor-Bendixson, see Sec. 4 . $\mathrm{mHC}=\mathrm{R}^{\mathrm{i}}+\mathrm{CB}^{\mathrm{i}}$
$\mathrm{CBL}^{\mathrm{i}}=\mathrm{CB}^{\mathrm{i}} \oplus \mathrm{GL}^{\mathrm{i}}$
$\mathrm{KM}=\mathrm{CB}^{\mathrm{i}} \oplus \mathrm{SL}^{\mathrm{i}}$
(gd) $\quad(A \rightarrow B) \vee(B \rightarrow A)$
$L C=I P C+(g d)$
$.3 \quad \square(\bullet A \rightarrow B) \vee \square(\odot B \rightarrow A)$
(ver) $\square A$
$\square A \quad \operatorname{Ver}^{\mathrm{i}}=\mathrm{K}^{\mathrm{i}} \oplus($ ver $)=\mathrm{K}^{\mathrm{i}} \oplus\left(\begin{array}{c}\text { (boxbot }) \\ \text { (boxbot })\end{array}\right.$
(nnv) $\neg \neg \square \perp$
$\mathrm{NNV}^{\mathrm{i}}=\mathrm{K}^{\mathrm{i}} \oplus(\mathrm{nnv})$
(nv) $\neg \square \perp$
$\mathrm{NV}^{\mathrm{i}}=\mathrm{K}^{\mathrm{i}} \oplus(\mathrm{nv})$ 


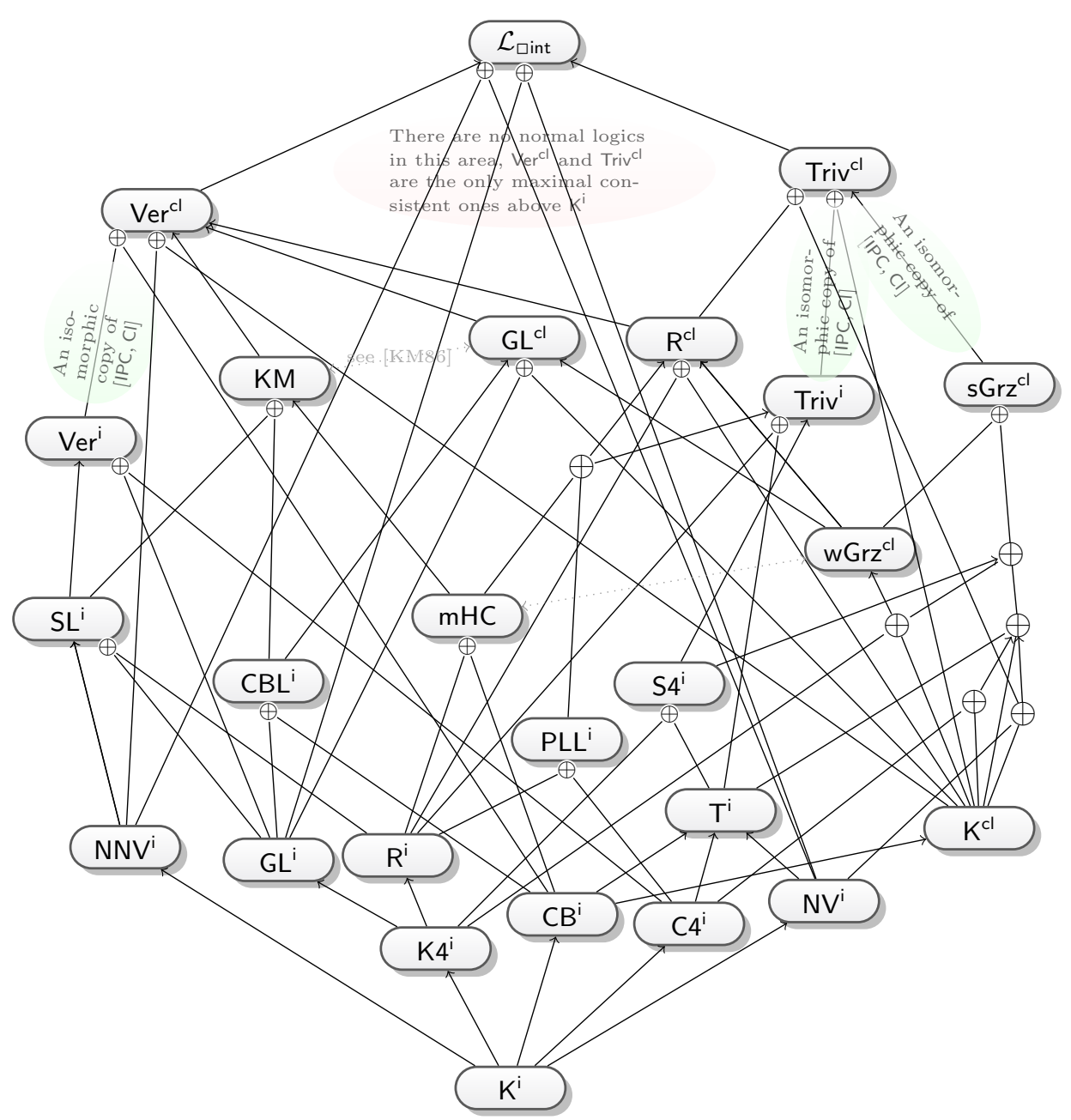

Figure 1: Relationships between systems introduced in Table 2 Ordinary arrows correspond to inclusion between systems. Lines joined by $\oplus$ denote joins: a join of two logics is of course the smallest normal modal logic containing both. Remember however that not all joins are shown, especially in case of joins with weak systems like $\mathrm{K}^{\mathrm{i}}$ or NNVi. For more on $\mathrm{wGrz}^{\mathrm{cl}}$, see Esa06 and also Lit07 and further references therein. The observation that $\operatorname{Triv}^{\mathrm{cl}}$ and $\mathrm{Ver}^{\mathrm{cl}}$ are the only maximal consistent logics in $\mathrm{K}^{\mathrm{i}}$ seems to have been made first by Vakarelov in Vak81. Wolter Wol97 is one of most advanced studies of the lattice of extensions of $\mathrm{K}^{\mathrm{i}}$; in particular, it solves a number of problem posed in Vak81 and investigates the counterparts of $\Gamma^{\mathrm{cl}}$ above $\mathrm{K}^{\mathrm{i}}$ for any given $\Gamma$. Finally, Wolter and Zakharyaschev WZ97 WZ98 show how to reinterpret this lattice as the lattice of extensions of a certain bimodal classical logic. This is in many ways the most general of Blok-Esakia type results as will be discussed later in Section 4.3 
4. $\mathrm{K}^{\mathrm{i}} \oplus(\mathrm{ufp})=\odot(B \leftrightarrow A[B / p]) \rightarrow(\odot(C \leftrightarrow A[C / p]) \rightarrow(B \leftrightarrow C))$

5. $\mathrm{K}^{\mathrm{i}} \oplus$ (henk) $=\odot(A \leftrightarrow \square A) \rightarrow A$

A variable $p \in \Sigma$ is $\square$-guarded in $A \in \mathcal{L}_{\square \text { int }}$ if all its occurrences are within the scope of $\square$. This notion will be used repeatedly in connection with $G L^{i}$ and its extensions.

Remark 3. Equalities in Table 2 and joins in Figure 1 should be in fact treated as a large lemma on interderivability for a number of intuitionistic normal modal axioms. Of particular interest for this chapter are: the derivability of ( $\mathrm{r}$ ) from (slöb), which mirrors derivability of (trns) from (wlöb); interderivability between (glb) and (slöb); equivalence between either of these and the conjunction of (wlöb) and ( $r$ ); two different ways of axiomatizing $\mathrm{CB}^{\mathrm{i}}$ by using (next) and (derv). All these statements are made assuming $\mathrm{K}^{\mathrm{i}}$.

All normal $\square$ int-logics as defined above - more precisely, their associated global consequence relations - are strongly finitely algebraizable; see standard references like BP89; Fon06; FJP03; FJP09: Ras74 for basic notions of algebraic logic and a more detailed discussion. Strong finite algebraizability also applies to normal logics in any fragment of $\square$ int containing $\rightarrow$. This is due to the fact that all these systems are implicative logics in the sense of Rasiowa Ras74. Given a normal logic $\Gamma$, I will call the corresponding class of algebras obtained via the algebraization process $\Gamma$-algebras, e.g., $\mathrm{K}^{\mathrm{i}}$-algebras, $\mathrm{GL}^{\mathrm{i}}$-algebras, $\mathrm{wGrz}^{\mathrm{cl}}$-algebras etc. $\mathrm{K}^{\mathrm{i}}$-algebras are obviously special cases of HAOs - Heyting algebras with operators - namely HAOs with a single unary operator. Recall that an operator on a Heyting algebra is an operation preserving $\top$ and finite meets. An operator on a Heyting algebra which turns it into a $\Gamma$-algebra will be called a $\Gamma$-operator.

Finally, recall that for any algebra $\mathfrak{H}$, a $\mathfrak{H}$-polynomial is a term in the similarity type of $\mathfrak{H}$ enriched with a separate constant for each element of $\mathfrak{H}$ [BS81, Definition 13.3]. In my notation for polynomials, I will not distinguish between an element of $\mathfrak{H}$ and its corresponding constant. Moreover, I will use elements of $\Sigma$ (i.e., propositional variables) as variables of polynomials, consistent with the general policy of blurring the distinction between logical formulas and algebraic terms. The notion of $\square$-guardedness for polynomials will be used in the same way as for formulas.

\subsection{Relational Semantics}

It is pretty obvious what should be the ingredients of a Kripke frame (a relational structure) for intuitionistic normal modal logic: it should be of the form $(W, \unlhd, \prec)$, where $\unlhd$ is a poset order used to interpret intuitionistic connectives, whereas $\prec$ is the modal accessibility relation used to interpret $\square$. Valuations of propositional variables should be defined as as $V: \Sigma \rightarrow \mathrm{Up}_{\unlhd}(W)$ and the inductive extension to intuitionistic connectives using $\unlhd$ is standard. However, once we set to provide a precise interpretation of modalities, there are choices to be made. Here is what Sim94, Section 3.3] — one of most comprehensive overviews - says on the subject:

One could take also the usual satisfaction clauses for the modalities in modal models ... However, an essential feature of intuitionistic models is the monotonicity lemma ... If the standard satisfaction clauses for the modalities are used then the monotonicity lemma does not hold. There are two possible remedies. (a) One is to modify the satisfaction clauses. This might be a reasonable thing to do, for 
one might wish to use the partial order to give a more intuitionistic reading of the modalities. (b) The other remedy is to impose conditions on models that ensure that the monotonicity lemma does hold.

The monotonicity lemma referred to in the above is of course the fact that the denotation of any intuitionistic formula in any Kripke model is upward closed. The two solutions lead to two alternative satisfaction definitions:

- $\square$ (a) $A:=\{w \in W \mid \forall v, x .(w \unlhd v$ and $v \prec x$ implies $x \in A)\}$

- $\square_{(\mathbf{b})} A:=\{w \in W \mid \forall x .(w \prec x$ implies $x \in A)\}$

By definition, $\square_{(\mathbf{a})} A \in \mathrm{Up}_{\triangleleft}(W)$ for any $A \subseteq W$. This, however, is easily seen to fail in general for (b), even for some $A \in \mathrm{Up}_{\triangleleft}(W)$. This is precisely while we need to impose additional conditions on the interaction of $\unlhd$ and $\prec$ :

Lemma $4([\overline{\mathrm{BD} 84}])$. For any $(W, \unlhd, \prec)$, the condition

$$
\text { for any } A \in \mathrm{Up}_{\unlhd}(W), \square_{(\mathbf{b})} A \in \mathrm{Up}_{\unlhd}(W)
$$

(i.e., $\mathrm{Up}_{\unlhd}(W)$ is a subalgebra of $\mathrm{Pow}(W)$ also with respect to $\left.\square_{(\mathbf{b})}\right)$ is satisfied iff the following condition holds:

$$
\unlhd ; \prec \subseteq \prec ; \unlhd
$$

Curiously, condition (1), even though isolated in [BD84] and discussed in references such as [Sim94], does not seem to appear too often in intuitionistic modal literature. Most references, even those which adopt interpretation (b), assume conditions stronger than (1). Goldblatt Gol81 requires

$$
\unlhd ; \prec \subseteq \prec
$$

but in most of intuitionistic modal logic literature (see, e.g., [Sot84; WZ97; WZ98]) we have still stronger

$$
\unlhd ; \prec ; \unlhd=\prec
$$

Obviously, (3) implies (2) and (2) implies (1), as $\unlhd$ is a partial order. But as noted in BD84, Gol81

$$
\prec ; \unlhd=\prec
$$

(i.e., for any $x, \prec^{-1}[x]$ is upward closed) cannot be forced by any axiom, as any frame $(W, \unlhd, \prec)$ can be shown to be semantically equivalent to $\left(W, \unlhd, \prec{ }^{\prime}\right)$ satisfying (4) by putting $\prec{ }^{\prime}=\prec ; \unlhd$.

In presence of (4), (1) implies (3) and hence all these conditions become equivalent. Thus, we can follow the others and assume (3), but from a logical point view, only (1) would be necessary.

Remark 5. A justification for adopting the strongest condition (3) is provided by completeness/canonicity result, for which the earliest references seem to be [BD84; Sot84]: when we define $\prec$ between prime filters of a Heyting algebra with a normal $\square$ in a standard way, the resulting Kripke frame will satisfy (3). This provides a justification of (3) and a bridge between algebraic and Kripke semantics, i.e., a completeness result for $\mathrm{K}^{\mathrm{i}}$ wrt Kripke frames satisfying (3) with $\square$ read as dictated by (b). 
Remark 6. What is the connection with (a)-semantics? It is easy to see that (a) reading of $\square$ wrt $\prec$ yields the same operator on $\mathrm{Up}_{\triangleleft}(W)$ as $(\mathbf{b})$-reading wrt $\prec$ modified to satisfy (2): that is, the closure of $\prec$ under pre-composition with $\unlhd$. Note here that in order to obtain a relation satisfying (3), one needs to close $\prec$ under both pre-and post-composition with $\unlhd$.

Thus, as stated in $\operatorname{Sim94}$

it turns out that both semantics induce the same intuitionistic modal logic. ... Only when the $\diamond$ connective is added do the differences in the semantics become apparent.

The reason why (a) is often seen in the literature (see Sim94 and references therein and also, e.g, AMPR01; PS86]) is that it becomes pretty natural when intuitionistic modal logic is considered as a fragment of intuitionistic predicate logic - and, in close connection, when one wants to interpret $\diamond$ using the same relation as for $\square$. However, this is not what we are interested in here. Thus, in what follows, I stick to (b) reading of modality (henceforth (b) is dropped as a subscript) and impose condition (3) on all frames. This is justified not only by Remark 5 above, but also by the fact that intuitionistic modal semantics in practically all references relevant for further discussion satisfied (3) anyway. Let us summarize the discussion above with the following definition:

A Kripke frame or a relational structure is of the form $(W, \unlhd, \prec)$, where

- $\unlhd$ is a poset order used to interpret intuitionistic connectives

- $\prec$ is the modal accessibility relation used to interpret $\square$ and

- $\unlhd ; \prec \subseteq \prec$, where ; is relation composition

A valuation is a mapping $V: \Sigma \rightarrow \mathrm{Up}_{\unlhd}(W)$, where $\mathrm{Up}_{\unlhd}(W)$ is the Heyting algebra of upward closed sets of $W$ and the inductive extension to $\mathcal{L}_{\square \text { int }} \Sigma$ is standard.

Remark 7. When at least one of lattice connectives is removed, the situation at first sight appears more complicated. While the papers proving the separation property of IPC [Hor62; Mil10; Pra65] showed that its reducts remain complete wrt relational semantics, a Stone-type representation theorem for arbitrary algebras would seem more problematic; the one for Heyting algebras relies crucially on the fact that they have distributive lattice reducts. However, a series of papers beginning with [Köh81] and finishing with [BJ13] established that Brouwerian semilattices enjoy in fact Stone-, Priestley-and Esakia-type dualities. 
Remark 8. It is worth discussing whether we can reconstruct this relational semantics from coalgebraic point of view, just like in the classical case. The relationship between the category of Heyting algebras (HA) and the category of posets with bounded morphisms (say, PABM) is the same as in the case of boolean algebras and sets (dual adjunction, restricting to equivalence in the finite case). Kripke frames for classical modal logic can be thought as coalgebras for covariant powerset functor. The counterpart of that functor in $\mathrm{PABM}$ is the covariant upset functor $\mathrm{Up}_{\unlhd}(\cdot)$, which sends $(W, \unlhd)$ to $\left(\mathrm{Up}_{\unlhd}(W), \supseteq\right)$ and $\mathrm{Up}_{\unlhd}(f)(A)=f[A]$. Then if we attempt to define relational structures as PABMmorphisms, i.e., bounded morphisms from $(W, \unlhd)$ to $\left(\mathrm{Up}_{\unlhd}(W), \supseteq\right)$, the very choice of codomain of this morphism forces (4) and the forth condition of bounded morphism becomes precisely (2), so altogether we get just (3). However, the back condition seems to impose a rather special condition on $\prec:$ for any $x \in W$ and any upward closed $A \subseteq \prec{ }^{-1}[x]$, there is $y$ s.t. $x \unlhd y$ and $\prec^{-1}[y]=A$. There are many natural examples of structures satisfying (3) where this condition would fail and in general I do not know how many logics would be possibly complete with respect to frames of this kind. It is not necessarily harmless for some of the axioms considered here. However, when we can forget about the back condition for morphisms (i.e., in case of modalities over distributive lattices rather than over Heyting algebras), (3) emerges as the natural basic semantic condition also from the coalgebraic point of view.

Table 3 lists semantic conditions corresponding to modal axioms. For $\mathrm{GL}^{\mathrm{i}}$ in particular, we have:

Theorem 9 ([Urs79] $)$. A structure $(W, \unlhd, \prec)$ validates $\mathrm{GL}^{\mathrm{i}}$ iff

- $\prec$ is transitive, i.e., $\prec ; \prec \subseteq \prec$ and

- $\prec$ is $\mathrm{Up}_{\unlhd}(W)$-Noetherian: for any $A \in \mathrm{Up}_{\unlhd}(W)$, if $A \neq W$, then there is $w \in \square A-A$, i.e., a $\square$-maximal non-A point

Proof (sketch). We show only the "if" direction. Assuming (wlöb) fails under a valuation $V$, take $B$ to be the extension of (wlöb) under $V$ and show that $\square B \subseteq B$. This means that $W-B$ witnesses the failure of Noetherianity.

Note that Theorem 9 together with the $(r)$ row of Table 3 provides also the condition for $\mathrm{SL}^{\mathrm{i}}$ : as already stated in Table 2 and Figure 1 , $\mathrm{SL}^{\mathrm{i}}$ is obtained as $\mathrm{GL}^{\mathrm{i}} \oplus(\mathrm{r})$, so one simply takes the conjunction of the two conditions.

Urs79 provides an interesting motivation for this semantics of $\mathrm{GL}^{i}$ in terms of projects and streamlines in a research. It also provides other important results, such as the finite model property and decidability.

\section{The Fixpoint Theorem}

Theorem 2 above gave us several equivalent axiomatizations of $\mathrm{GL}^{\mathrm{i}}$. In particular, (ufp) forces uniqueness of fixed points.

Definition 10. Let $B$ be a formula of $\mathcal{L}_{\text {Dint }}$ (its denotation in a given algebra and under a given valuation). $B$ is a fixed point of (the term function associated with) $A \in \mathcal{L}_{\square \text { int }}$ relative 
Table 3: Semantic counterparts of intuitionistic modal axioms. See, e.g., Doš85; Sot84 WZ97; WZ98 for more. $\Delta:=\{(w, w) \mid w \in W\}$ and $\triangleleft:=\unlhd-\Delta$, i.e., it is the strict version of the intuitionistic poset order. For $R \in\{\prec, \unlhd\}, V \subseteq W$, $V^{R}:=\{w \in W \mid \exists v \in V . v R w\}$ and $V_{\downarrow}^{R}:=\{w \in W \mid \exists v \in V . w R v\}$

Axiom Semantic condition

(cl) $\unlhd=\Delta$

(r) $\prec \subseteq \unlhd$

(next) $\triangleleft \subseteq \prec$

(nnv) $\quad\left(W-W^{\prec} \downarrow\right) \unlhd \downarrow=W$
Axiom Semantic condition Axiom Semantic condition

(trns) $\prec ; \prec \subseteq \prec$

(bind) $\prec \subseteq \prec ; \prec$

(refl) $\unlhd \subseteq \prec$

(ver) $\prec=\emptyset$

(nv) $\quad W^{\prec} \downarrow=W$

to $p$ in a given normal logic $\Gamma$ (here always an extension of $\mathrm{GL}^{\mathrm{i}}$ ) if $B \leftrightarrow A[B / p] \in \Gamma$ and $p$ does not occur in $B$.

According to Smo79, the fact that (ufp) holds in $\mathrm{GL}^{\mathrm{cl}}$ was discovered independently by Bernardi, Sambin and de Jongh. We know thus that, surprisingly, in $G^{i}$ a syntactic fixed point of an expression is unique up to equivalence whenever it exists; same applies to all of its extensions, such as $\mathrm{SL}^{\mathrm{i}}$ or KM. But do they exist at all? An even more amazing fact is that they not only do exist - under the assumption of $\square$-guardedness on $p$ - but are effectively computable. This is guaranteed by the following algebraic (or propositional, if one prefers) variant of Gödel's Diagonalization Lemma. Sambin [Sam76] proved it for GL ${ }^{i}$ itself and de Jongh proved it for $\mathrm{GL}^{\mathrm{cl}}$ building on an earlier result by Smorynski, another proof being found soon afterwards by Boolos; the reader is referred to [Boo93; BS91; Mur14; Smo79] for more on its history and the connection with Gödel's result:

Theorem 11 (Diagonalization). For any $A$ and $p$, there exists a constructively obtained formula $\operatorname{diag}_{p} A$ s.t.

1. $\operatorname{diag}_{p} A \leftrightarrow B \in \mathrm{GL}^{\mathrm{i}}$, where $B$ is obtained from $A$ by replacing all occurrences of $p$ under $\square$ by $\operatorname{diag}_{p} A$

2. $A$ and $\operatorname{diag}_{p} A$ have provably the same fixed points with respect to $p$, that is, for any $C$ not containing $p$ we have

$$
\ominus(C \leftrightarrow A[C / p]) \leftrightarrow \odot\left(C \leftrightarrow \operatorname{diag}_{p} A[C / p]\right) \in \mathrm{GL}^{\mathrm{i}}
$$

Clearly, if $p$ is $\square$-guarded in $A$, then $B$ in the first clause is precisely $A\left[\operatorname{diag}_{p} A / p\right]$ and $\operatorname{diag}_{p} A$ does not contain $p$, hence being trivially its own fixed point wrt $p$. Thus, in such a situation $\operatorname{diag}_{p} A$ itself is also the unique fixed-point of $A$ with respect to $p$ !

Proof of Theorem 11, sketch. We only give a sketch of how $\operatorname{diag}_{p} A$ is constructed. Any formula $A(p, \bar{q}) \in \mathcal{L}_{\square \text { int }}$ in variables $p, \bar{q} \in \Sigma$ can be written as $B\left(\square C_{1}(p, \bar{q}), \ldots, \square C_{k}(p, \bar{q}), p, \bar{q}\right)$, where $B \in \mathcal{L}_{\text {int }}$ (i.e., is a formula without $\square$ ) and $\bar{C} \in \mathcal{L}_{\square \text { int }}$. Clearly, if $k=0$, then $A$ itself belongs to $\mathcal{L}_{\text {int }}$ and, in particular, there are no occurrences of $p$ under $\square$. Hence we can take $\operatorname{diag}_{p} A$ to be $A$ itself. Otherwise, the proof can be conducted by induction on $k$, as we already have the base step. For any $i \leq k$, set

$$
A_{i}:=B\left(\square C_{1}(p, \bar{q}), \ldots, \square C_{i-1}(p, \bar{q}), \top, \square C_{i+1}(p, \bar{q}), \ldots, \square C_{k}(p, \bar{q}), p, \bar{q}\right) .
$$


By definition, the inductive hypothesis applies to $A_{i}$. Now we set

$$
\operatorname{diag}_{p} A:=B\left(\square C_{1}\left(\operatorname{diag}_{p} A_{1} / p, \bar{q}\right), \ldots, \square C_{k}\left(\operatorname{diag}_{p} A_{k} / p, \bar{q}\right), p, \bar{q}\right) .
$$

Remark 12. In fact, extensions of $\mathrm{SL}^{\mathrm{i}}$-in particular $\mathrm{KM}$ - allow a much simpler proof of Theorem 11 and a much simpler algorithm for computing these fixpoints: it is enough to substitute $T$ for $p$. This follows already from observations made by Smorynski in [Smo79, Lemma 2.3] and has been discussed explicitly in [JV95. Propositions 4.2-4.6]. De Jongh and Visser describe $\mathrm{SL}^{\mathrm{i}}$ as a kind of Kindergarten Theory in which all the well-known syntactical results of Provability Logic have extremely simple versions.

Remark 13. It is known that at least in the case of $\mathrm{GL}^{\mathrm{cl}}$ a non-constructive and nonexplict form of Theorem 11 can be obtained already from uniqueness of fixed-points combined with the Beth definability theorem for $\mathrm{GL}^{\mathrm{cl}}$, see, e.g., [Boo93; Smo79] for more information. However, as should be clear from the discussion below, the very fact that fixed points are obtained explicitly and constructively seems an advantage not to be given up lightly.

Theorem 11 has a nice algebraic corollary. I present it here as a more general version of Esa06, Proposition 3].

Theorem 14. A $\mathrm{K}^{\mathrm{i}}{ }^{\mathrm{i}}$-algebra $\mathfrak{H}$ is a $\mathrm{GL}^{\mathrm{i}}$-algebra iff every $\mathfrak{H}$-polynomial $t(p)$ in one $\square$-guarded variable $p \in \Sigma$ has a fixed point.

Proof. The "only if" direction. This is a direct corollary of Theorem 11.

The "if" direction. Given any $\mathrm{h} \in \mathfrak{H}$, consider the polynomial $t(p)=\square p \rightarrow \mathrm{h}$. As $p$ is $\square$-guarded in it, it has a fixed point $i_{h} \in \mathfrak{H}$; that is, $i_{h}=\square i_{h} \rightarrow h$. By the fact that $\rightarrow$ is conjugate (or residual) to $\wedge$, one half of this equality is equivalent to $i_{h} \wedge \square i_{h} \leq h$. On the other hand, $\mathrm{h} \leq \square \mathrm{i}_{\mathrm{h}} \rightarrow \mathrm{h}=\mathrm{i}_{\mathrm{h}}$ by general implication laws. Taken together, these two inequalities imply $\mathrm{i}_{\mathrm{h}} \wedge \square \mathrm{i}_{\mathrm{h}}=\mathrm{h} \wedge \square \mathrm{h}$ : the $\leq$ direction from the first inequality, normality and (trns), the $\geq$ direction from the second inequality and monotonicity of $\square$. Using normality again, we get $\square \mathrm{i}_{\mathrm{h}} \wedge \square \square \mathrm{i}_{\mathrm{h}}=\square \mathrm{h} \wedge \square \square \mathrm{h}$ and using (trns) again, we arrive at $\square \mathrm{i}_{\mathrm{h}}=\square \mathrm{h}$. Then we get $\square(\square h \rightarrow h)=\square\left(\square i_{h} \rightarrow h\right)=\square i_{h}=\square h$. As $h \in \mathfrak{H}$ was chosen arbitrarily, we have that $\mathfrak{H}$ is a $G^{i}$-algebra.

There is an analogy between the above result and alternative axiomatizations for $\mathrm{GL}^{\mathrm{i}}$ presented in Urs79.

\section{Corollary 15.}

- $A \mathrm{R}^{\mathrm{i}}$-algebra $\mathfrak{H}$ is a $\mathrm{SL}^{\mathrm{i}}$-algebra iff every $\mathfrak{H}$-polynomial $t(p)$ in one $\square$-guarded variable $p \in \Sigma$ has a fixed point.

- Esa06, Proposition 3] A mHC-algebra $\mathfrak{H}$ is a $\mathrm{KM}$-algebra iff every $\mathfrak{H}$-polynomial $t(p)$ in one $\square$-guarded variable $p \in \Sigma$ has a fixed point. 
As stated above, Theorem 11 occurred first as an algebraization of Gödel's Diagonalization Lemma. While the connection between $\mathrm{GL}^{\mathrm{i}}$ and Heyting Arithmetic HA is not as tight as the one between $\mathrm{GL}^{\mathrm{cl}}$ and Peano Arithmetic PA established by the completeness result of Solovay [Sol76] (see also Boo93, Chapter 9]), [Sam76] notes that Theorem 11 yields a counterpart of the Diagonalization Lemma for any intuitionistic first-order theory with a canonical derivability predicate, including obviously HA. At any rate, the relevance of fixpoint results for Löb-like logics is not limited to arithmetic.

Remark 16. It is worth mentioning here that-unlike the case of PA - the search for a complete axiomatization of the provability logic of $\mathrm{HA}$ is not over yet; [Iem01] gives a fascinating account. Regarding the arithmetical interpretation of $\mathrm{SL}^{\mathrm{i}}$, see the discussion of $\mathrm{HA}^{*}$ in [JV95, Sec. 4-5].

To begin with, in the classical case one can use Theorem 11 to show that explicit smallest or greatest fixed-point operators are eliminable over $\mathrm{GL}^{\mathrm{cl}}$. In other words, adding $\mu$ or $\nu$ does not increase the expressive power of the classical modal logic of transitive and conversely wellfounded structures; see AF09; Ben06; Vis05]. Note that this includes all correctly formed expressions with $\mu$, without assuming that all occurrences of $p$ are $\square$-guarded: as usual, they only have to be positive. CFL10, Section 3] discusses an application in the context of expressivity of navigational fragments of XML query languages.

While I am not aware of an exact analogue of the results in AF09 Ben06 Vis05 in the intuitionistic context ${ }_{1}^{1}$ Löb-like modalities - more specifically, variants of systems GL $^{i}$ and $\mathrm{SL}^{\mathrm{i}}$ - have recently become rather popular in type theory. Examples include:

- modality for recursion Nak00, Nak01

- approximation modality AMRV07

- guardedness type constructor AM13

- next-step modality/next clock tick KB11a; KB11b

- later operator BT09; BM13; BMSS12; JTS12,

One of reasons is precisely that such modalities guarantee existence and uniqueness of fixed-points of suitably guarded type expressions. However, the modal spadework of 1970's seems rarely acknowledged. In [Nak00, which may be credited with introducing intuitionistic Löb-like modalities to the attention of this community, we find the following statement:

Similar results concerning the existence of fixed points of proper type expressions ... could historically go back to the fixed point theorem of the logic of provability ... The difference is that our logic is intuitionistic, and fixed points are treated as sets of realizers [the emphasis is mine-T.L.].

This formulation suggests that Nakano was not aware that the intuitionistic fixed-point theorem had been already proved in Sam76], not to mention improvements possible above $\mathrm{SL}^{\mathrm{i}}$ (cf. Remark 12). The only related references quoted in Nak00 focus on classical GL ${ }^{\mathrm{cl}}$ e.g, Boo93 - and in later papers even these are omitted. A valuable part of the logical tradition seems lost this way. Let us see what insights can be found in the work of the Tbilisi school.

\footnotetext{
${ }^{1}$ It is a theme I am presently collaborating on with Albert Visser.
} 


\section{Leo Esakia and Extensions of $\mathrm{mHC}$}

\section{1 $\mathrm{mHC}$ and Topological Derivative}

Leo Esakia and collaborators devoted special attention to the system $\mathrm{mHC}$ and its extensions. Esa06 is an excellent overview. The abbreviation mHC stood for modalized Heyting calculus. The reader may find the name surprising; after all, many natural intuitionistic modal systems are not subsystems of $\mathrm{mHC}$. Esakia [Esa06] was perfectly aware of that:

The postulate $(r)$ is not typical, while the postulate (derv) stresses even more "nonstandardness" of the chosen basic system $\mathrm{mHC}$ and of its extension $\mathrm{KM}$, which enables one to draw a conventional "demarcation line" between $\mathrm{mHC}$ and the standard intuitionistic modal logics.

Remark 17. Both axioms seem "nonstandard" mostly if one focuses on these intuitionistic modal logics which are obtained from popular classical systems. It is enough to look at Table 3 to realize why it must be so: (derv) is trivially derivable in $\mathrm{K}^{\mathrm{cl}}$ (its consequent being a classical tautology), while the combination of $(\mathrm{cl})$ and $(\mathrm{r})$ yields that $\prec \subseteq \Delta$. That is, the only classical frames for $\mathrm{R}^{\mathrm{cl}}$ are disjoint unions of reflexive and irreflexive points. However, ( $\mathrm{r}$ ) is nowhere as pathological in a properly intuitionistic setting. There are many references on systems different from $\mathrm{mHC}$ and $\mathrm{KM}$ where nevertheless $(\mathrm{r})$ is still derivable or even explicitly included as an axiom. A short and inexhaustive list includes [Aba07; Acz01; BBP98; BMSS12; Cur52; FM97; GA08; GP06; Gol81; Gol10; KB11b; MP08; Nak00; Nak011. They can be split roughly into two main groups. The first onee.g., [BMSS12; KB11b; Nak00; Nak01]-concerns $\mathrm{SL}^{\mathrm{i}}$ and has already been mentioned in Section 3. The second one-e.g., (Acz01; BBP98; Cur52; FM97; GA08; GP06; Gol81; Gol10]-concerns the system which is denoted here as $\mathrm{PLL}^{\mathrm{i}}$ (a.k.a. CL, see [BBP98]). [Gol03, Section 7.6] and [Gol10] are good if incomplete overviews of most relevant papers on this system - the most important omissions being perhaps [PD01, Section 7] and [Aba07; GP06]. See also [MP08] for a discussion of type systems with $\mathrm{R}^{\mathrm{i}}$ modalities from programmer's point of view.

Esa06] gives the following reasons for the importance of $\mathrm{mHC}$ :

- Its connection to KM: $\mathrm{mHC}$ is KM minus the Löb axiom (slöb). Note that (wlöb) and (slöb) are equivalent in the presence of $(r)$

- The connection with intuitionistic temporal logic "Always \& Before" possessing rich expressive possibilities

- The fact that $\mathrm{mHC}$ can be obtained as a fragment of QINT (or, in Esakia's notation, QHC) - quantified intuitionistic propositional calculus. This is similar to the encoding of $\mathrm{mHC}$ in the internal language of a topos, see the last point

- The topological connection with Cantor's scattered spaces, notions of the limit and isolated point. This will be discussed at length in this section

- Finally, as mentioned above, $\mathrm{mHC}$ turns out to be a natural fragment of the topos logic. This last point builds on all the preceding ones and will be discussed in Sec. 5 
As we can see in Table 3 , the conditions on the accessibility relation $\prec$ imposed by the axioms of $\mathrm{mHC}$ - the combination of $(\mathrm{r})$ and (derv) — is $\triangleleft \subseteq \prec \subseteq \unlhd$. A natural question to ask is whether it is possible to enforce syntactically that $\prec$ is even more closely determined by $\unlhd$ as one of the two borderline cases, i.e., either $\prec=\unlhd$ or $\prec=\triangleleft$.

For $\prec=\unlhd$, the answer is obviously positive. This is achieved precisely by the axioms of the logic Trivi, strengthening (derv) to (refl). In fact, this is a semantic counterpart of an observation in Esa06 that enriching any Heyting algebra with a trivial operator $\square^{\text {Triv }^{i}} x:=x$ yields an $\mathrm{mHC}$-algebra. Note here that whenever $\square$ is an $\mathrm{mHC}$ - or even $\mathrm{R}^{\mathrm{i}}$-operator, its associated $\square$ is a Triv'-operator.

For $\prec=\triangleleft$, the answer is obviously negative. Irreflexivity is a typical example of a condition which cannot be defined by any purely modal axiom, see BdV01. Here is perhaps the most natural proof.

Example 18. Consider a frame for $\mathrm{mHC}$ defined as $(\omega, \unlhd, \triangleleft)$ where $\unlhd$ is the natural order $\leq$ on $\omega$. The dual algebra contains as a subalgebra the two-element Boolean algebra with $\square \perp=\perp$, which is the dual of a single $\prec$-reflexive point. Hence, no modal axiom can define the class of $\prec$-irreflexive frames over the class of frames for $\mathrm{mHC}$.

However, an "irreflexive" mHC-operator is clearly definable on any Heyting algebra obtained as the dual of an intuitionistic Kripke frame $(W, \unlhd)$ : for any $A \in \mathrm{Up}_{\unlhd}(W)$, we take $* A:=$ $\{w \in W \mid\{w\} \unlhd \uparrow-\{w\} \subseteq A\}$; again, see Table 3 for notation. It is straightforward to note that for any $w \in W, w \in * A$ iff for any $B \in \mathrm{Up}_{\unlhd}(W), w \in(B \rightarrow A) \cup B$. This observation actually explains the shape of axiom (derv). Hence, given any complete Heyting algebra $\mathfrak{H}$, define its point-free coderivative EJP00, $\operatorname{Sim} 82$, Sim14 as $* \mathrm{~h}:=\bigwedge_{\mathrm{i} \in \mathfrak{H}}(\mathrm{i} \vee(\mathrm{i} \rightarrow \mathrm{h}))$.

Proposition 19. For any complete Heyting algebra, its point-free coderivative is an $\mathrm{mHC}$ operator.

Proof. A rather easy exercise for the reader; can be also extracted from the proof of Esa06, Proposition 5].

There is a slightly different description of $*$. Take a Heyting algebra $\mathfrak{H}$ and $\mathrm{h}, \mathrm{i} \in \mathfrak{H}$ s.t. $\mathrm{i} \leq \mathrm{h}$. $\mathrm{h}$ is $\mathrm{i}$-dense or dense in $[\mathrm{i}, \top]$ if for any $\mathrm{j} \in \mathfrak{H}$, we get that $\mathrm{h} \wedge \mathrm{j}=\mathrm{i}$ implies $\mathrm{j}=\mathrm{i}$. Note that the standard topological notion of density can be considered a special case: an open set is topologically dense iff it is $\perp$-dense in the Heyting algebra of open sets of the space. The following was observed, e.g., in EJP00]:

Fact 20. For any Heyting algebra $\mathfrak{H}$ and any $\mathrm{h} \geq \mathrm{i} \in \mathfrak{H}, \mathrm{h}$ is $\mathrm{i}$-dense iff there exists $\mathrm{j} \in \mathfrak{H}$ s.t. $\mathrm{h}=\mathrm{j} \vee(\mathrm{j} \rightarrow \mathrm{i})$.

Corollary 21. For any complete Heyting algebra $\mathfrak{H}$ and any $\mathrm{i} \in \mathfrak{H}$,

$$
* \mathrm{i}=\bigwedge\{\mathrm{h} \in \mathfrak{H} \mid \mathrm{h} \geq \mathrm{i} \text { and } \mathrm{h} \text { is } \mathrm{i} \text {-dense }\} .
$$

Why coderivative? The reader is referred to a detailed account by Simmons Sim14. Briefly, recall that in topology the Cantor-Bendixson derivative of a set $A$ is the set of those $x$ whose every neighbourhood contains a point of $A$ other than $x$; the dual operator (hence co-derivative) consists of those $x$ which have an open neighbourhood entirely contained in $A \cup\{x\}$ Esa06. As it turns out, this indeed coincides with $*$ for practically all sensible topological spaces: 
Theorem 22 (Simmons). For any $T_{0}$-space, its co-derivative operator coincides with the point-free coderivative * on the Heyting algebra of open sets.

Proof. For * defined as in Corollary 21, this was proved in Sim82.

Obviously, as any intuitionistic Kripke frame with the Alexandroff topology given by $\mathrm{Up}_{\triangleleft}(W)$ is $T_{0}$, we get that $*$ coincides with the dual of topological derivative of this topology. It is, in fact, easier to prove directly than by Simmons' result.

Remark 23. One observation from [Sim82] is worth quoting here:

for non-T-Spaces the usual definition of isolated point does not quite capture the intended notion

and hence for arbitrary spaces, the point-free definition of derivative given by * seems in fact more adequate than the standard one. The reader can verify this by extending the definition of intuitionistic Kripke frames to qosets rather than just posets and checking how both notions would fare in such a setting.

\subsection{KM and Scatteredness}

A complete Heyting algebra will be called scattered if its coderivative $*$ is not only an $\mathrm{mHC}$ operator, but a KM-operator. Recall Corollary 15 as an algebraic characterization of such a situation.

\section{Proposition 24.}

- For any topological space, its point-free coderivative is a $\mathrm{KM}$-operator if the space is scattered in the usual sense: that is, if each non-empty subset has an isolated point

- For any $T_{0}$ topological space, its point-free coderivative is a $\mathrm{KM}$-operator only if the space is scattered

Proof. A non- $T_{0}$-space can never be scattered, and for $T_{0}$-spaces, the point-free coderivative coincides with ordinary one as stated in Theorem 22. The remaining calculations are an exercise in point-set topology; in fact, the point-set part of this result has been shown first by Kuznetsov Kuz79; Mur14. One can use an alternative characterization of scatteredness here: for any open set $A$ distinct from the whole space, $* A-A$ is non-empty.

Let us summarize some of the results above:

Corollary 25. A topological space $\mathfrak{T}$ is scattered iff in the complete Heyting algebra of open sets of $\mathfrak{T}$, every polynomial in one $*$-guarded variable has a fixed point.

Corollary 26. The following are equivalent for any $(W, \unlhd, \prec)$ :

- $\prec=\triangleleft$ and the Alexandroff topology $\left(W, \mathrm{Up}_{\unlhd}(W)\right)$ is scattered

- $(W, \unlhd, \prec)$ is a frame for $\mathrm{KM}$

- $\prec=\triangleleft$ and it contains no infinite ascending chains 
Proof. We only need to prove the equivalence of the last two conditions. $\triangleleft \subseteq \prec$ is, as observed, enforced by $\mathrm{mHC}$. Theorem 9 gives the corresponding semantic condition for (wlöb). Quite obviously, $\mathrm{Up}_{\unlhd}(W)$-Noetherianity forces irreflexivity of $\prec$. Thus, whenever $(W, \unlhd, \prec$ ) is a frame for $\mathrm{KM}$ (that is, the join of $\mathrm{mHC}$ and $\mathrm{GL}^{\mathrm{i}}$ ), we have $\prec=\triangleleft$. Moreover, rewriting the condition of $\mathrm{Up}_{\triangleleft}(W)$-Noetherianity for $\mathrm{mHC}$-frames, we obtain that for any $A \in \mathrm{Up}_{\triangleleft}(W)-\{W\}$, there is $w \in A^{\triangleleft} \uparrow-A$. Rewriting further, we obtain that any $B \neq \emptyset$ s.t. $B=B \unlhd \downarrow$ has a maximal element wrt $\unlhd$. But this means that any nonempty subset of $W$ has a maximal $\unlhd$-element.

\subsection{Completeness, Lattice Isomorphisms and Bimodal Translations}

Two important kinds of results have been missing from this overview so far. First, while I discussed Kripke correspondence for modal logics (Table 3, Theorem 9 and Corollary 26), I have not discussed completeness. Second, I have not said much about lattices of extensions of Löb-like logics and their relatives - in particular, about generalizations of the Blok-Esakia Theorem.

This section fixes both oversights. Rather than using original proofs of Kuznetsov, Muravitsky (for $\mathrm{KM}$ ) and Esakia (for $\mathrm{mHC}$ ), we are going to use corollaries of Wolter and Zakharyaschev's results on bimodal translations [WZ97; WZ98], briefly discussed also in [WZ14, Section 4]. Their techniques allow to interpret intuitionistic modal logics as fragments of classical polymodal ones (cf. the discussion of implict vs. explicit epistemics in Ben91]). In the case of the Blok-Esakia theorem for $\mathrm{mHC}$, we will be able to see why axioms of $\mathrm{mHC}$ and $\mathrm{wGrz}^{\mathrm{cl}}$ have to look the way they look in order to allow the classical counterpart to be unimodal rather than polymodal, as it happens in the more general framework of [WZ97; WZ98].

Take the bimodal language $\mathcal{L}_{\mathrm{i}, \mathrm{m}}$ with operators [i] and [m]. For any formula $A \in \mathcal{L}_{\square \text { int }}$, I will write $A_{[\mathrm{i}]}$ (respectively $A_{[\mathrm{m}]}$ ) for $A$ with all occurrences of $\square$ replaced with [i] (respectively $[\mathrm{m}]) .[\mathrm{m}]$ is the default counterpart of the original modality $\square$ and [i] encodes the intuitionistic poset order, hence the notation. ${ }^{2}$ The logic $S 4_{i, m}^{c l}$ is the normal logic axiomatized by the following axioms: $(\mathrm{cl}),(\mathrm{nrm})_{[\mathrm{i}]},(\mathrm{refl})_{[\mathrm{i}]},(\text { trns })_{[\mathrm{i}]}$ and $(\mathrm{nrm})_{[\mathrm{m}]}$; in other words, it is what modal logicians would describe as the fusion of $\mathrm{S}_{\mathbf{i}}^{\mathrm{cl}}$ and $\mathrm{K}_{\mathbf{m}}^{\mathrm{cl}}$. The $\operatorname{logic} \mathrm{S} 4 \mathrm{M}_{\mathrm{i}, \mathrm{m}}^{\mathrm{cl}}$ is $\mathrm{S} 4_{\mathrm{i}, \mathrm{m}}^{\mathrm{cl}}$ extended with

$$
(\operatorname{mix}): \quad[\mathrm{m}] A \leftrightarrow[\mathrm{i}][\mathrm{m}][\mathrm{i}] A .
$$

The logic $\mathrm{sGrzM}_{\mathrm{i}, \mathrm{m}}^{\mathrm{cl}}$ is $\mathrm{S}_{\mathrm{i}, \mathrm{m}}^{\mathrm{cl}}$ extended with $(\mathrm{mix})$ and $(\mathrm{sgrz})_{[\mathrm{i}]}$. The translation $b: \mathcal{L}_{\square \text { int }} \rightarrow$ $\mathcal{L}_{\mathrm{i}, \mathrm{m}}$ prefixes every subformula in $(\cdot)_{[\mathrm{m}]}$ with [i]. Of course, many occurrences of $[i]$ in the translation $b A$ can be removed relative to logics defined above:

Fact 27. The following equivalences belong to $\mathrm{S}_{\mathrm{i}, \mathrm{m}}^{\mathrm{cl}}: b A \leftrightarrow[\mathrm{i}] \mathrm{b} A, b(A \wedge B) \leftrightarrow(b A \wedge b B)$, $b(A \vee B) \leftrightarrow(b A \vee b B)$; in $\mathrm{S}_{4} \mathrm{M}_{\mathrm{i}, \mathrm{m}}^{\mathrm{cl}}$, we moreover have $b(\square A) \leftrightarrow[\mathrm{m}] \mathrm{b} A$.

For any intuitionistic normal modal $\operatorname{logic} \Gamma \supseteq \mathrm{S}_{\mathrm{i}, \mathrm{m}}^{\mathrm{cl}}$ and any bimodal normal logic $\Delta \subseteq \mathcal{L}_{\mathrm{i}, \mathrm{m}}$, let

- $\tau^{\mathrm{mix}} \Gamma:=\mathrm{S}_{4} \mathrm{M}_{\mathrm{i}, \mathrm{m}}^{\mathrm{cl}} \oplus\{b A \mid A \in \Gamma\}$

- $\sigma^{\text {mix }} \Gamma:=\tau^{\text {mix }} \Gamma \oplus(\operatorname{sgrz})_{[\mathrm{i}]}$

- $\rho_{\text {in }} \Delta:=\left\{A \in \mathcal{L}_{\text {ロint }} \mid b A \in \Delta\right\}$

${ }^{2}$ The reader has to be warned that the notation in this section differs somewhat from that in references like WZ97, WZ98, WZ14. 
$\Delta$ is $a \mathcal{L}_{\mathrm{i}, \mathrm{m}}$-companion of $\Gamma$ if for any $A \in \mathcal{L}_{\text {口int }}, A \in \Gamma$ iff $b A \in \Delta$, i.e., iff $\rho_{\text {in }} \Delta=\Gamma$.

Theorem 28 (|WZ97; WZ98, WZ14] $)$. Let $\Delta \supseteq \mathrm{S}_{\mathrm{i}, \mathrm{m}}^{\mathrm{cl}}$ be a normal bimodal logic and $\Gamma \subseteq \mathcal{L}_{\square \mathrm{int}}$ be an intuitionistic normal logic. Then

(A) $\rho_{\text {in }} \Delta$ is an intuitionistic normal modal logic

(B) $\tau^{\text {mix }} \Gamma$ and $\sigma^{\text {mix }} \Gamma$ are, respectively, the smallest and the greatest $\mathcal{L}_{\mathrm{i}, \mathrm{m}}$-companions of $\Gamma$ containing (mix)

(C) $\rho_{\text {in }}$ preserves decidability, Kripke completeness and the finite model property. If (mix) $\in$ $\Delta, \rho_{\text {in }}$ also preserves canonicity

(D) $\tau^{\text {mix }}$ preserves canonicity

(E) $\sigma^{\text {mix }}$ preserves the finite model property

(F) $\sigma^{\text {mix }}$ is an isomorphism from the lattice of normal extensions of $\mathrm{K}^{\mathrm{i}}$ onto the lattice of normal extensions of $\mathrm{s} \mathrm{GrzM}_{\mathrm{i}, \mathrm{m}}^{\mathrm{cl}}$

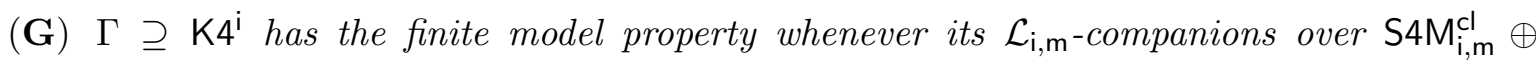
(trns) $[\mathrm{m}]$ include a canonical subframe logic

Proof. (A) can be easily proved from Fact 27; note that we need the assumption we are above $\mathrm{S}_{\mathrm{i}, \mathrm{m}}^{\mathrm{cl}}$. (B) is a consequence of Theorem 27 in WZ97. (C), (D) and (E) are consequences of Proposition 29 and Theorem 30 in [WZ97] and Theorems 11 and 12 in [WZ98.(F) is a consequence of Corollary 28 in [WZ97]. (G) is a consequence of Corollary 18 in [WZ98].

WZ97; WZ98 illustrate on many examples how powerful these results are. As it turns out, they also have corollaries of immediate interest for us.

Corollary 29. $\mathrm{mHC}$ is canonical and has the finite model property.

Proof. First, note that

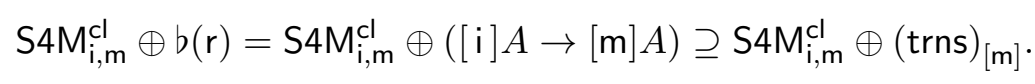

Clearly, [i] $A \rightarrow[\mathrm{m}] A$ is a Sahlqvist formula with an universal FO counterpart. Furthermore, $S 4 M_{i, m}^{c l} \oplus b($ derv $)$ is the same logic as the extension of $S 4 M_{i, m}^{c l}$ with

$$
[\mathrm{m}] B \wedge\langle\mathrm{i}\rangle C \rightarrow[\mathrm{i}](\langle\mathrm{i}\rangle C \vee[\mathrm{i}] B) .
$$

The latter is a simple Sahlqvist implication (cf. e.g., BdV01, Definition 3.47]). Applying the algorithm in the proof of Theorem 3.49 in BdV01] and doing some FO-preprocessing, we get an universal formula

$$
\forall y, z, w \cdot(x \unlhd y \wedge x \unlhd z \rightarrow(z \unlhd y \vee(z \unlhd w \rightarrow x \prec w)))
$$

(where $\unlhd$ is the accessibility relation corresponding to [i] and $\prec$ is the accessibility relation corresponding to $[\mathrm{m}])$. Thus, $\tau^{\mathrm{mix}} \mathrm{mHC}$ is a canonical subframe logic over $\mathrm{S}_{4} \mathrm{M}_{\mathrm{i}, \mathrm{m}}^{\mathrm{cl}} \oplus(\mathrm{trns})_{[\mathrm{m}]}$. Now, canonicity of $\mathrm{mHC}$ follows from $(\mathbf{C})$ and the fmp from $(\mathbf{G})$ of Theorem 28 . 
Remark 30. It is worth noting that the semantic counterpart of (next) from Table 3, i.e., $\triangleleft \subseteq \prec$ is equivalent to (6) above. For one direction, substitute $x=y$ in (6) and use poset properties. For the other direction, note that whenever $x \unlhd y$ and $x \unlhd z$ but $\neg(z \unlhd y)$, then $x \triangleleft z$, ergo $x \prec z$. Now whenever $z \unlhd w$, we can use the interaction between $\prec$ and $\unlhd$ as expressed by (mix) (in fact, even a weaker axiom would do).

Canonicity of $\mathrm{mHC}$ has been noted, e.g., in [Esa06; Gol81]. I was unable to locate references where the finite model property has been explicitly claimed. The following corollary can appear more surprising, as bimodal logics over $\mathcal{L}_{\mathrm{i}, \mathrm{m}}$ do not even occur in its statement.

Corollary 31. The lattice of normal extensions of $\mathrm{mHC}$ is isomorphic to the lattice of normal extensions of $\mathrm{wGrz}^{\mathrm{cl}}$. The sublattice of normal extensions of $\mathrm{KM}$ is isomorphic to the lattice of normal extensions of $\mathrm{GL}^{\mathrm{cl}}$.

Proof. The heart of the proof is to notice that $[\mathrm{i}] A \leftrightarrow[\mathrm{m}] A \wedge A$ and $(\mathrm{grz})_{[\mathrm{m}]}$ are derivable in $\sigma^{\text {mix }} \mathrm{mHC}$; in fact, these two formulas axiomatize this logic over $\mathcal{L}_{\mathrm{i}, \mathrm{m}} \oplus(\mathrm{trns})_{[\mathrm{m}]}$. Let us derive the first of them. For convenience, we will do it in the algebraic setting:

$$
\begin{aligned}
{[\mathrm{m}] A \wedge A \wedge\langle\mathrm{i}\rangle \neg A } & \leq & & \text { by }(\text { refl })_{[\mathrm{i}]} \\
{[\mathrm{m}] A \wedge\langle\mathrm{i}\rangle(A \wedge\langle\mathrm{i}\rangle \neg A) } & \leq & & \text { by }(5) \\
{[\mathrm{i}](\langle\mathrm{i}\rangle(A \wedge\langle\mathrm{i}\rangle \neg A) \vee[\mathrm{i}] A) } & \leq & & \text { by }(\text { refl })_{[\mathrm{i}]} \\
{[\mathrm{i}](\langle\mathrm{i}\rangle(A \wedge\langle\mathrm{i}\rangle \neg A) \vee A) } & = & & \\
{[\mathrm{i}]([\mathrm{i}](A \rightarrow[\mathrm{i}] A) \rightarrow A) } & \leq[\mathrm{i}] A & & \text { by }(\mathrm{grz})_{[\mathrm{i}]} .
\end{aligned}
$$

We get that $\sigma^{\text {mix }} \mathrm{mHC}$ is just a notational variant of $\sigma^{\mathrm{mix}} \mathrm{wGrz}^{\mathrm{cl}}$, with $[\mathrm{m}]$ being $\square$ and [i] being $\odot$. This yields the first statement by clause $(\mathbf{F})$ of Theorem 28 . For the second, it is enough to add the observation that over $\sigma^{\text {mix }} \mathrm{mHC}$, adding $b($ slöb) is equivalent to adding $(\mathrm{wlöb})_{[\mathrm{m}]}$.

The second statement of Corollary 31 above was first proved by Kuznetsov and Muravitsky in mid-1980's, see [KM86; Mur14]. The first statement was announced in [Esa06 as follows:

Finally let us note that ... the lattice Lat $(\mathrm{mHC})$ of all extensions of $\mathrm{mHC}$ is isomorphic to the lattice Lat(K4.Grz) of all normal extensions of the modal system K4.Grz. However, a proof of this result requires additional considerations as the above algebraic machinery does not suffice for it.

It seems that the proof has not been published so far.

Corollary 32 (Gol81; KM86; Mur81]). KM has the finite model property.

Proof. The proof of Corollary 31 has established that $\sigma^{\text {mix }} \mathrm{KM}$ is just a notational variant of $\mathrm{GL}^{\mathrm{cl}}$, with $[\mathrm{m}]$ being $\square$ and $[\mathrm{i}]$ being $\odot$. Now use clause $(\mathbf{C})$ of Theorem 28 and the finite model property for $\mathrm{GL}^{\mathrm{cl}}$ (see, e.g., BdV01; Boo93; $\mathrm{CZ97}$; Fin85; Mos07 for references). 
Remark 33. Note that we could also prove Corollary29 in an analogous way to Corollary 32. using the fmp of $\mathrm{wGrz}^{\mathrm{cl}}$ established explicitly by Amerbauer [Ame96]. The latter is actually a direct consequence of $\mathrm{wGrz}^{\mathrm{cl}}$ being a transitive subframe logic [Fin85; Lit07]. However, I believe that the proof of Corollary 29 provided above has some additional value: we obtained a convenient form of b(derv) - which we actually used in the proof of Corollary 31 -together with its FO translation, which also provides some additional insight, as discussed in Remark 30.

Remark 34. It could be an interesting exercise-and very much in the spirit of the Tbilisi school - to show that the above-discussed results of [WZ97; WZ98; WZ14] survive when the base bimodal logic is weakened from $\mathrm{S}_{\mathrm{i}, \mathrm{m}}^{\mathrm{cl}}$ to the fusion of $\mathrm{K}_{\mathrm{i}}^{\mathrm{cl}} \oplus(A \wedge[\mathrm{i}] A \rightarrow$ $[\mathrm{i}][\mathrm{i}] A)$ with $\mathrm{K}_{\mathbf{m}}^{\mathrm{cl}}$ and the translation $b$ is modified to $b^{*}$ replacing every subformula $A$ with $b^{*} A \wedge[i] b^{*} A$. On the other hand, it is not obvious how much generality would be really gained in this way. Note that using Wolter and Zakharyaschev's original results we were able to investigate lattices of logics which are not extensions of $\mathrm{S}^{\mathrm{cl}}$, such as $\mathrm{wGrz}^{\mathrm{cl}}$ in Corollary 31 above.

\section{Scattered Toposes}

We are ready to discuss the topos of trees of [BMSS12], scattered toposes of [EJP00] and the relationship between fixpoint results in both papers.

\subsection{Preliminaries on Topos Logic}

Just like Section 4.3 assumed certain familiarity with technicalities of modal logic, this section in turn assumes some familiarity with basics of category theory - mostly with the notions of a ccc (cartesian closed category), a functor and a natural transformation. Those readers who know more than that, in particular understand well the internal logic of a topos, can probably skip this subsection. Due to obvious space constraints, the presentation has to be rather abstract and example-free; see Gol06, Joh02, MM92 for more examples and motivation. Furthermore, like any presentation of topos theory by logicians and for logicians, it can be accused of neglecting spatial intuitions. See, e.g., [McL90] for a passionate polemic with the view that toposes were invented to generalize set-theoretical foundations of mathematics $3^{3}$ Nevertheless, applications of toposes in fields like algebraic geometry or foundations of physics or their actual historical origins are not directly relevant here. My aim is a minimalist presentation focusing on the contrast between the logic of a topos and that of a ccc, but also making clear how the Beth-Kripke-Joyal semantics is related to more familiar ones for the intuitionistic predicate logic. Of all accounts in the literature, the one in [LS86] is probably closest to this goal.

\footnotetext{
${ }^{3}$ Speaking of McL90, footnote 4 provides an argument that the plural form intended by Grothendieck was toposes rather than topoi. I stick to the same convention, also because - as a quick Google search showsthe form toposes is used mostly by mathematicians, whereas topoi seems prevalent for unrelated notions in the humanities. Besides, this was the form used by Leo, Mamuka and Dito.
} 
Let $\mathscr{E}$ be a ccc with the terminal object 1 and for any $Y \in \mathscr{E}$, let fin! $Y$ be the unique element of $\mathscr{E}[Y, \mathbf{1}]$. I use the obvious notation for (finite) products, coproducts (whenever they exist, but in a topos they always do), their associated morphisms and I denote the ccc evaluation mapping $B^{A} \times A \rightarrow B$ as eval en $_{B}$. Recall that $\mathscr{E}$ is an elementary topos if there exists an object $\Omega \in \mathscr{E}$ s.t. $(\Omega, \mathbf{1} \stackrel{\text { true }}{\rightarrow} \Omega)$ is a subobject classifier, i.e., for any monic (left-cancellable morphism) $Y \stackrel{f}{\longmapsto} X$ there exists exactly one mapping $X \stackrel{\chi_{f}}{\rightarrow} \Omega$ s.t. we have a pullback diagram:

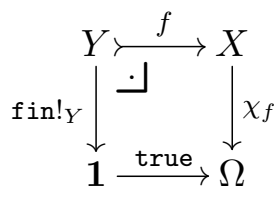

As observed by C. Juul Mikkelsen, this definition already implies that $\mathscr{E}$ is bicartesian closed, where the latter notion is defined as in, e.g., [LS86]; see [Gol06, Section 4.3] for references.

Before we proceed with formal definitions, some general discussion can be helpful. In every category, topos or not, (equivalence classes of) monics into $X$ are abstract counterparts of subsets of $X$; in fact, they are called subobjects of $X$, just like morphisms $\mathbf{1} \rightarrow X$ are global elements of $X$. Global elements (or equivalence classes thereof) can be considered as special cases of subobjects: think of the usual identification of an element $x \in X$ with the subset

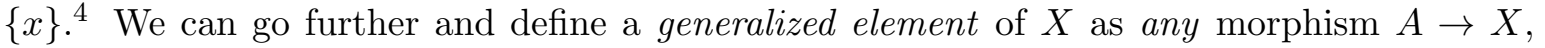
which is then called A-based or defined over A. See [MM92, Section V] for a lucid and brief discussion of those notions.

In particular, the global elements of $\Omega$ can be identified with logical constants, $X$-based generalized elements of $\Omega$ with predicates over $X$ (i.e., formulas with a single free variable from $X)$ and $n$-ary propositional connectives with morphisms $\Omega^{n} \rightarrow \Omega$. Set false $:=\chi_{\text {fin! }}$, $\neg:=\chi_{\text {false }}, \wedge:=\chi_{\langle\text {true }, \text { true }\rangle}$ and $\vee:=\chi_{\left[\left\langle\operatorname{true}_{\Omega}, \text { id }_{\Omega}\right\rangle,\left\langle\text { id }_{\Omega}, \text { true }_{\Omega}\right\rangle\right]}$. Recall that for any $X \in \mathscr{E}$, true $_{X}$ stands for true $\circ$ fin! $X$ and $\mathrm{eq}_{X}$ stands for $\chi_{\left\langle\mathrm{id}_{X}, \mathrm{id}_{X}\right\rangle}$. The latter allows to define internal equality for generalized elements of type $X$ as $\sigma \approx \tau:=\mathrm{eq}_{X} \circ\langle\sigma, \tau\rangle$. That is, if $A \stackrel{\sigma}{\rightarrow} X$ and $B \stackrel{\tau}{\rightarrow} X$ are generalized elements of $X$, then $\sigma \approx \tau$ is a generalized element of $\Omega$ defined over $A \times B$. For $\Omega$, we can define not only eq ${ }_{\Omega}$, but also $\operatorname{leq}_{\Omega}$ as the equalizer of $\Omega \times \Omega \stackrel{\wedge}{\stackrel{\longrightarrow}{\Pi_{1}}} \Omega$. Implication, the only remaining intuitionistic connective, can be now defined as $\rightarrow:=\chi_{\operatorname{leq}_{\Omega}}$.

Thus, in toposes one can reduce reasoning about the poset of subobjects of any given object $X \in \mathscr{E}$ (in fact, whenever $\mathscr{E}$ is a topos, this poset is always a lattice and even a Heyting algebra - see [MM92, Theorem IV.8.1]) to reasoning about $\mathscr{E}[X, \Omega]$ and further still to reasoning about an internal Heyting algebra provided by a suitable exponential object. What this means is: in any category, monics into $X$ have a natural preorder defined as $f \subseteq g$ if $f$ factors through $g$, i.e., there is a morphism $h$ s.t. $f=g \circ h$. Dividing by equivalence classes with respect to $\subseteq$, we get a category-theoretic generalization of the poset of subsets of $X$ ordered by inclusion. In general, without understanding the global structure of $\mathscr{E}$, we are not likely to learn much about these posets of subobjects. But in a topos, the poset of subobjects of $X$ is isomorphic to something more tangible: namely, to $\mathscr{E}[X, \Omega]$, i.e., $X$-based generalized elements of $\Omega$. Think of the usual identification of subsets of $X$ with elements of

\footnotetext{
${ }^{4}$ Note that toposes very rarely happen to mimic sets in having enough global elements to determine all subobjects; such special toposes are called well-pointed.
} 
$2^{X}$. Here is also where first- and higher-order aspects of the internal logic come into play.

If $\mathscr{E}$ is a category with products, a power object of $X \in \mathscr{E}$ is a pair $\left(\mathcal{P} X, \ominus{ }_{X} \stackrel{\ni}{\longrightarrow} \mathcal{P} X \times X\right)$ s.t. for any $(Y, R \succ \stackrel{r}{\longrightarrow} Y \times X)$ there exists exactly one $Y \stackrel{f_{r}}{\longrightarrow} \mathcal{P} X$ for which there is a pullback

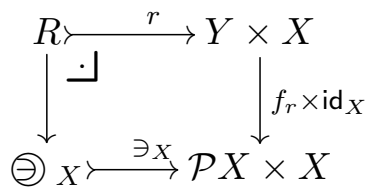

As shown, e.g., in Gol06, Theorem 4.7.1], in any topos we can take $\mathcal{P} X$ to be $\Omega^{X}$ and the subobject $\ominus \vartheta_{X} \stackrel{\ni_{X}}{\longrightarrow} \Omega^{X} \times X$ can be obtained by pulling back true along $\Omega^{X} \times X \stackrel{\text { eval }_{X}, \Omega}{\rightarrow} \Omega$. Thus, we see that in a topos, the notions of power object, subobject classifier and exponential object are indeed well-matched and we can define the membership predicate $\sigma \in \tau$ for a pair of generalized elements $(A \stackrel{\sigma}{\rightarrow} X, B \stackrel{\tau}{\rightarrow} \mathcal{P} X)$ as eval $X, \Omega \circ\langle\tau, \sigma\rangle$. We are now ready for a single definition formalizing the whole discussion above and more (see [MM92, Sec. VI.5-7] and also Cro93; LS86):

Definition 35 (The Mitchell-Bènabou languague). Consider a topos $\mathscr{E}$. The collection of ground types and the signature of the Mitchell-Bènabou language of $\mathscr{E}$ are defined, respectively, as

$$
\begin{aligned}
\operatorname{Ground}(\mathscr{E}) & :=\{\underline{E} \mid E \in \mathscr{E}\} \\
\operatorname{Sg}(\mathscr{E}) & :=\left\{\underline{f}: \underline{F_{1}}, \ldots, \underline{F_{n}} \rightarrow \underline{E} \mid f \in \mathscr{E}\left[F_{1} \times \cdots \times F_{n}, E\right]\right\}
\end{aligned}
$$

(instead of $\underline{k}: \underline{1} \rightarrow \underline{E}$ I will write $\underline{k}: \underline{E}$ ) and the full collection of types Types $(\mathscr{E})$ is $A, B::=\underline{E}|\underline{\mathbf{1}}| \underline{\Omega}|A \underline{\times} B| B^{A}$ where $\underline{E} \in \operatorname{Ground}(\mathscr{E})$. $\underline{\Omega}^{A}$ can be also written as $\underline{\mathcal{P}} A$. Fix, moreover, a supply of term variables $x, y, z \cdots \in \mathrm{tVar}$. The collection of terms Terms $(\mathscr{E})$ over $\operatorname{Sg}(\mathscr{E})$ is defined as

$$
M, N::=x|\underline{f} \bar{M}| \underline{\text { fin! }}|M \approx N| \underline{\langle M, N\rangle}\left|\underline{\Pi_{1}} M\right| \underline{\Pi_{2}} M|\lambda x: A \cdot M| M^{\underline{2}} N
$$

where $x \in \mathrm{tVar}$ and $f \in \mathrm{Sg}(\mathscr{E})$ is of suitable arity. The typing rules and some standard abbreviations (including all logical connectives) of the language are defined in Table 4. Interpretation of types, contexts and terms-in-context in $\mathscr{E}$ is given in Table 5 .

Definition 36 (Forcing for an elementary topos). Assume $\Gamma=x_{1}: \underline{F_{1}}, \ldots, x_{n}: \underline{F_{n}}$ and $\Gamma \vdash \phi: \underline{\Omega} . B y\left\{\left\langle x_{1} \ldots x_{n}\right\rangle \in F_{1} \times \cdots \times F_{n} \mid \phi\right\}$, I will denote the pullback of the following diagram:

$$
\begin{gathered}
{[\Gamma]} \\
1 \stackrel{\text { true }}{\longrightarrow} \stackrel{\mid[\Gamma \vdash \phi: \Omega]}{\Omega}
\end{gathered}
$$

Now for $F \stackrel{f_{7}}{\rightarrow} F_{1}, \ldots, F \stackrel{f_{n}}{\rightarrow} F_{n}$ write $F, f_{1}, \ldots, f_{n} \Vdash \phi$ if $F \stackrel{\left\langle f_{1}, \ldots, f_{n}\right\rangle}{\rightarrow}[\Gamma]$ factors through $\left\{\left\langle x_{1} \ldots x_{n}\right\rangle \in F_{1} \times \cdots \times F_{n} \mid \phi\right\} \rightarrow[\Gamma]$. In what follows, $f_{1} \circ g, \ldots, f_{n} \circ g$ will be denoted by $\bar{f} \circ g$. Moreover, let $[\Gamma \vdash \phi: \underline{\Omega}]=$ true $_{[\Gamma]}$ be written as $\Gamma \vDash_{\mathscr{E}} \phi$.

Fact 37. 


$$
\begin{aligned}
& \overline{\Gamma, x: A \vdash x: A} \quad \frac{\Gamma \vdash M: A \quad \Gamma \vdash N: A}{\Gamma \vdash M \approx N: \underline{\Omega}} \quad \overline{\Gamma \vdash \underline{\text { fin! }: \underline{\mathbf{1}}}} \quad \frac{\Gamma \vdash M: A \Gamma \vdash N: B}{\Gamma \vdash \underline{\langle M, N\rangle: A \times B}} \\
& \frac{\Gamma, x: A \vdash M: B}{\Gamma \vdash \lambda x: A . M: B^{A}} \quad \frac{\Gamma \vdash M: B^{A} \quad \Gamma \vdash N: A}{\Gamma \vdash M^{2} N: B} \quad \frac{\Gamma \vdash M: A \underline{\times} B}{\Gamma \vdash \underline{\Pi_{1}} M: A} \quad \frac{\Gamma \vdash M: A \underline{\times} B}{\Gamma \vdash \underline{\Pi}_{2} M: B} \\
& \frac{\underline{f}: \underline{F_{1}}, \ldots, \underline{F_{n}} \rightarrow \underline{E} \in \operatorname{Sg}(\mathscr{E}) \quad \Gamma \vdash M_{1}: \underline{F_{1}} \ldots \Gamma \vdash M_{n}: \underline{F_{n}}}{\Gamma \vdash \underline{f} M_{1} \ldots M_{n}: \underline{E}}
\end{aligned}
$$

$\underline{\text { true }}:=\underline{\text { fin! }} \approx \underline{\text { fin! }}$

$\forall x: A . \phi:=\lambda x: A . \phi \approx \lambda x: A$.true

$\exists x: A . \phi:=\forall t: \underline{\Omega} .((\forall x: A . \phi \rightsquigarrow t) \rightsquigarrow t)$

$\phi \vee \psi:=\forall t: \underline{\Omega} .((\phi \rightsquigarrow t) \wedge(\psi \rightsquigarrow t) \rightsquigarrow t)$ $\phi \wedge \psi:=\langle\phi, \psi\rangle \approx\langle\underline{\text { true }}, \underline{\text { true }}\rangle$

$\phi \rightsquigarrow \psi:=\phi \wedge \bar{\psi} \approx \phi$

$\underline{\text { false }}:=\forall t: \underline{\Omega} . t$

$\neg \phi:=\phi \rightsquigarrow$ false

Table 4: Typing rules and defined abbreviations of the Mitchell-Bènabou language of $\mathscr{E}$

$$
\begin{aligned}
& {[\underline{E}]:=E \quad[\underline{1}]:=1 \quad[\underline{\Omega}]:=\Omega \quad[A \underline{\times} B]:=[A] \times[B] \quad\left[B^{A}\right]:=[B]^{[A]}} \\
& {\left[x_{1}: A_{1}, \ldots, x_{n}: A_{n}\right]:=\left[A_{1} \rrbracket \times \cdots \times\left[A_{n}\right]\right.} \\
& \overline{[\Gamma, x: A \vdash x: A]=\pi:[\Gamma] \times[A] \rightarrow[A]} \quad \overline{[\Gamma \vdash \text { fin! }: \underline{1}]=\mathrm{fin} ![\Gamma]} \\
& \frac{f \in \mathscr{E}\left[A_{1} \times \cdots \times A_{n}, B\right]\left[\Gamma \vdash M_{1}: A_{1}\right]=\sigma_{1}:[\Gamma] \rightarrow\left[A_{1}\right] \ldots\left[\Gamma \vdash M_{n}: A_{n}\right]=\sigma_{n}:[\Gamma] \rightarrow\left[A_{n}\right]}{\left[\Gamma \vdash f M_{1} \ldots M_{n}: B\right]:=f \circ\left\langle\sigma_{1}, \ldots, \sigma_{n}\right\rangle:[\Gamma] \rightarrow B} \\
& {[\Gamma \vdash M: A]=\sigma:[\Gamma] \rightarrow[A] \quad[\Gamma \vdash N: A]=\tau:[\Gamma] \rightarrow[A]} \\
& {\left[\Gamma \vdash M \approx N: \underline{\Omega]}:=\chi_{\left\langle\mathrm{id}_{[\Gamma]}, \mathrm{id}_{[\Gamma]}\right\rangle} \circ\langle\sigma, \tau\rangle\right.} \\
& \frac{[\Gamma \vdash M: A]=\sigma:[\Gamma] \rightarrow[A] \quad[\Gamma \vdash N: B]=\tau:[\Gamma] \rightarrow[B]}{[\Gamma \vdash\langle M, N\rangle: A \underline{\times} B]:=\langle\sigma, \tau\rangle:[\Gamma] \rightarrow[A] \times[B]} \\
& \frac{[\Gamma \vdash M: A \times B]=\sigma:[\Gamma] \rightarrow[A] \times[B]}{\left[\Gamma \vdash \Pi_{1} M: A\right]:=\Pi_{1} \circ \sigma:[\Gamma] \rightarrow[A]} \quad \frac{[\Gamma \vdash M: A \times B]=\sigma:[\Gamma] \rightarrow[A] \times[B]}{\left[\Gamma \vdash \Pi_{2} M: B\right]:=\Pi_{2} \circ \sigma:[\Gamma] \rightarrow[B]} \\
& {[\Gamma, x: A \vdash M: B]=\sigma:[\Gamma] \times[A] \rightarrow[B]} \\
& {\left[\Gamma \vdash \lambda x: A . M: B^{A}\right]:=\text { curry }^{[\Gamma] \times[A],[B]}(\sigma):[\Gamma] \rightarrow[B]^{[A]}} \\
& {\left[\Gamma \vdash M: B^{A}\right]=\sigma:[\Gamma] \rightarrow[B]^{[A]} \quad[\Gamma \vdash N: A]=\tau:[\Gamma] \rightarrow[A]} \\
& {\left[\Gamma \vdash M^{2} N: B\right]:=\operatorname{eval}_{[A],[B]} \circ\langle\tau, \sigma\rangle}
\end{aligned}
$$

Table 5: Interpretation of types, contexts and terms-in-context 
- $F, \bar{f} \Vdash \phi$ iff $\left[\Gamma \vdash \phi: \underline{\Omega]} \circ \bar{f}=\operatorname{true}_{F}\right.$

- $\Gamma \vDash_{\mathscr{E}} \phi$ iff for any $F \stackrel{\bar{f}}{\rightarrow}[\Gamma]$, it holds that $F, \bar{f} \Vdash \phi$

The following result, which can be found as Theorem VI.6.1 in MM92 or Theorem II.8.4 in [LS86, connects the definition of forcing given above with more standard intuitionistic semantics:

Theorem 38 (Beth-Kripke-Joyal semantics in an elementary topos). Assume $F \stackrel{f_{1}}{\rightarrow} E_{1}, \ldots, F \stackrel{f_{n}}{\rightarrow}$ $E_{n}$ and $\Gamma=x_{1}: \underline{E}_{1} \ldots x_{n}: \underline{E}_{n}$.

- $F, \bar{f} \Vdash \phi \wedge \psi$ iff $F, \bar{f} \Vdash \phi$ and $F, \bar{f} \Vdash \psi$

- $F, \bar{f} \Vdash \phi \vee \psi$ iff there are arrows $G \stackrel{g}{\rightarrow} F$ and $H \stackrel{h}{\rightarrow} F$ s.t. $G+H \stackrel{[g, h]}{\rightarrow} F$ is epi, $G, \bar{f} \circ g \Vdash \phi$ and $H, \bar{f} \circ h \Vdash \psi$

- $F, \bar{f} \Vdash \phi \rightsquigarrow \psi$ iff for any $G \stackrel{g}{\rightarrow} F$ it holds that $G, \bar{f} \circ g \Vdash \psi$ whenever $G, \bar{f} \circ g \Vdash \phi$

- $F, \bar{f} \Vdash \neg \phi$ iff for any $G \stackrel{g}{\rightarrow} F$, it holds that $G \cong \mathbf{0}$ whenever $G, \bar{f} \circ g \Vdash \phi$

For the case of quantified formulas, note that $\Gamma \vdash \forall x_{n+1}: \underline{E}_{n+1} . \phi$ iff $\Gamma, x_{n+1}: \underline{E}_{n+1} \vdash \phi$. Same holds for $\Gamma \vdash \exists x_{n+1}: \underline{E}_{n+1}$. $\phi$. Then we have:

- $F, \bar{f} \Vdash \forall x_{n+1}: \underline{E}_{n+1} . \phi$ iff for every $G \stackrel{g}{\rightarrow} F$ and every $G \stackrel{g^{\prime}}{\rightarrow} E_{n+1}$ it holds that $G, f_{1}$ 。 $g, \ldots, f_{n} \circ g, g^{\prime} \Vdash \phi$

- $F, \bar{f} \Vdash \exists x_{n+1}: \underline{E}_{n+1} . \phi$ iff there exist $G \stackrel{g^{\prime}}{\rightarrow} E_{n+1}$ and an epi $G \stackrel{g}{\rightarrow} F$ s.t. $G, f_{1} \circ g, \ldots, f_{n} \circ$ $g, g^{\prime} \Vdash \phi$

- $F, \bar{f} \Vdash \sigma \approx \tau$ iff $[\Gamma \vdash \sigma: \underline{E}] \circ \bar{f}=[\Gamma \vdash \tau: \underline{E}] \circ \bar{f}$

The clauses for $\exists$ and $\vee$ above resemble those of intuitionistic Beth semantics. This is why "Beth-Kripke-Joyal" seems a more appropriate name in the general case of an arbitrary elementary topos; see, e.g., Gol06, Section 14.6]. However, when the topos happens to be the topos of presheaves, i.e., covariant functors into Set, on a given small category $\mathscr{R}$-in particular, a poset taken as a category - the definition of forcing can be significantly simplified ${ }^{5}$

Perhaps the most straightforward account of this simplification can be found in LS86. First, the clause for disjunction can be "kripkefied" for indecomposable objects and the clause for existential quantifiers - for projective ones [LS86, Proposition 8.7]. Second, the second clause of Fact 37 suggests that to check validity of a given judgement-in-context $\Gamma \vdash \phi: \underline{\Omega}$ in a topos, it is enough to restrict attention to those $F$ which belong to a generating set for a given topos. Third, by the Yoneda Lemma, in a topos of presheaves Set $^{\mathscr{R}}$ for an arbitrary small category $\mathscr{R}$, objects of the form $\operatorname{hom}_{\mathscr{R}}^{C}:=\mathscr{R}[C,-]$ for any given $C \in \mathscr{R}$ satisfy all these conditions: they are projective, indecomposable and do form a generating set. Moreover, also by the Yoneda Lemma, elements of $\mathbf{S e t}^{\mathscr{R}}\left[\operatorname{hom}_{\mathscr{R}}^{C}, F\right]$ are in $1-1$ correspondence with elements of $F(C)$ :

$$
\begin{gathered}
\operatorname{Set}^{\mathscr{R}}\left[\operatorname{hom}_{\mathscr{R}}^{C}, F\right] \ni f \rightarrow \breve{f}:=f_{C}\left(\operatorname{id}_{C}\right) \\
F(C) \times \operatorname{hom}_{\mathscr{R}}^{C} \ni(c, h) \rightarrow \hat{c}(h):=F h(c)
\end{gathered}
$$

\footnotetext{
${ }^{5}$ Reader should be warned that in most of categorical literature, presheaves are assumed to be contravariant, but see, e.g., Ghi89 for an example of the covariant convention.
} 
Note also that in clauses like the one for $\rightsquigarrow$, we can restrict attention to those $G \stackrel{g}{\rightarrow} F$ whose source $G$ lies in the generating set. In the case of $\operatorname{Set}^{\mathscr{R}}$, this means replacing $G \stackrel{g}{\rightarrow}$ hom $_{\mathscr{R}}^{C}$ with elements of $\operatorname{Set}^{\mathscr{R}}\left[\operatorname{hom}_{\mathscr{R}}^{D}, \operatorname{hom}_{\mathscr{R}}^{C}\right]$. But, by the Yoneda Lemma again, these can be replaced with arrows in $\mathscr{R}[C, D]$ (note the change of direction!).

Taking all this into account, we can obtain the following modified version of the semanticsthis time properly "Kripkean" (see [LS86, Proposition 9.3]).

Corollary 39 (Kripke-Joyal semantics in a topos of presheaves). Let $\mathscr{R}$ be a small category, $F_{1}, \ldots, F_{n} \in \operatorname{Set}^{\mathscr{R}}, C \in \mathscr{R}, c_{1} \in F_{1}(C), \ldots c_{n} \in F_{n}(C), \Gamma=c_{1}: \underline{F}_{1}, \ldots, c_{n}: \underline{F}_{n}$ and $\Gamma \vdash \phi: \underline{\Omega}$. Write $C, \bar{c} \Vdash \phi$ for $\operatorname{hom}_{\mathscr{R}}^{C}, \hat{c}_{1}, \ldots, \hat{c}_{n} \Vdash \phi$. Given any $f \in \mathscr{R}[\bar{C}, D]$, write $f(\bar{c})$ for $\hat{c}_{1}(f), \ldots, \hat{c}_{n}(f)$ - that is, $F f\left(c_{1}\right), \ldots, F f\left(c_{n}\right)$. Then we have:

- $C, \bar{c} \Vdash \phi \wedge \psi$ iff $C, \bar{c} \Vdash \phi$ and $C, \bar{c} \Vdash \psi$

- $C, \bar{c} \Vdash \phi \vee \psi$ iff $C, \bar{c} \Vdash \phi$ or $C, \bar{c} \Vdash \psi$

- $C, \bar{c} \Vdash \phi \rightsquigarrow \psi$ iff for any $f \in \mathscr{R}[C, D], D, f(\bar{c}) \Vdash \psi$ whenever $D, f(\bar{c}) \Vdash \phi$

- $C, \bar{c} \Vdash \neg \phi$ iff for any $f \in \mathscr{R}[C, D]$, it does not hold that $D, f(\bar{c}) \Vdash \phi$

- $C, \bar{c} \Vdash \forall x_{n+1}: \underline{F}_{n+1} . \phi$ iff for every $f \in \mathscr{R}[C, D]$ and $d \in F_{n+1}(D)$, it holds that $D, f(\bar{c}), d \Vdash \phi$

- $C, \bar{c} \Vdash \exists x_{n+1}: \underline{F}_{n+1} . \phi$ iff there exist $d \in F_{n+1}(C)$ s.t. $C, \bar{c}, d \Vdash \phi$

[Ghi89] uses toposes of presheaves as a generalization of Kripke semantics for the intuitionistic first-order logic to prove incompleteness results. Of numerous follow-ups of that work, let me just mention [NI97; Skv12]. Let us also note that the derivation of Corollary 39 from Theorem 38 takes a somewhat more roundabout route in [MM92]: toposes of presheaves are handled there as a subclass of toposes of sheaves on a site.

\subsection{Non-expansive Morphisms, Fixpoints and Scattered Toposes}

Let $\mathscr{E}$ be an elementary topos. Call an endomorphism $f \in \mathscr{E}[X, X]$ unchanging [EJP00] or non-expansive if

$$
\vDash_{\mathscr{E}} \forall x, y: \underline{X} \cdot(\underline{f} x \approx \underline{f} y \rightsquigarrow x \approx y) \rightsquigarrow x \approx y .
$$

As noted in EJP00, in a boolean setting non-expansive means just constant: negate the sentence and play with boolean laws. Obviously then a classical proof that a non-expansive endomorphism on a non-empty set has a unique fixed point does not carry much computational content. In a constructive setting, however, the situation is different.

Assume $\vdash \phi, \psi: \underline{\Omega}^{\underline{X}}$ and $f \in \mathscr{E}[X, X]$ and define:

$$
\begin{aligned}
\operatorname{SubTe}(\phi) & :=\forall x, y: \underline{X}\left(\phi^{2}-x \wedge \phi^{2} y \rightsquigarrow x \approx y\right) \\
\phi \subseteq \psi & :=\forall x: \underline{X} \cdot(\phi \underline{?}-x \rightsquigarrow \psi-x) \\
\operatorname{MaxST}(\phi) & :=\operatorname{SubTe}(\phi) \wedge \forall \alpha: \underline{\Omega^{2}} \underline{X} .(\operatorname{SubTe}(\alpha) \wedge \phi \subseteq \alpha \rightsquigarrow \alpha \subseteq \phi) \\
\operatorname{Non} \_\exp (\underline{f}) & :=\forall x, y: \underline{X} \cdot(\underline{f} x \approx \underline{f} y \rightsquigarrow x \approx y) \rightsquigarrow x \approx y \\
\text { fix_so }_{\underline{f}} & :=\lambda x: \underline{X} .(x \approx \underline{f} x)
\end{aligned}
$$

With this apparatus, we can state the main Theorem of Section 3 of [EJP00]: 
Theorem 40. Assume $\mathscr{E}$ is an elementary topos and $f \in \mathscr{E}[X, X]$. Then

$$
\vDash_{\mathscr{E}} \operatorname{Non} \_\exp (\underline{f}) \rightsquigarrow \operatorname{MaxST}\left(\text { fix } \_ \text {so }_{\underline{f}}\right) .
$$

Remark 41. A proof formalized in the Coq proof assistant is available from the author, see Remark 1. Those who would like to try a manual yet rigorous proof in the MitchellBènabou language should do first Exercise 5 in [LS86, p. 139] and then formalize the proof in [EJP00, p. 105] using all the abbreviations given above.

Remark 42. Some of derivations in [Esa98; EJP00], especially when fully formalized in a proof assistant, make explicit an interesting fact: certain axioms governing quantification which are not generally intutionistically valid (e.g., the Kuroda axiom and its generalization due to Casari), become valid in presence of a $\mathrm{KM}$ modality, even though these axioms do not involve any modalities at all in their statement. Some related observations are made by [Bir12] (a reference I became aware of having written the published version of this overview).

In words, this result says: the fixpoints of a non-expansive endomorphism form a maximal subterminal subobject ${ }^{6}$ The syntactic shape of SubTe $f$ easily suggests that subterminality is the internal counterpart of "being of cardinality at most one", i.e., uniqueness of fixpoints. However, the situation with existence is more complicated. First of all, toposes of presheaves can differ significantly from the topos of sets in having non-trivial objects with no global elements whatsoever. More importantly, even being inhabited is not enough to ensure maximal subterminal objects are global elements.

Example $43([\overline{E J P 00}])$. Consider the topos of presheaves on $(\omega+1, \unrhd)$, where $\unrhd$ is the converse of the standard ordinal order. Presheaf $X$ defined as $X(n)=n+1$ and $X(\omega)=\omega$ with $X(\beta \unrhd \alpha)(n)=\min (n, \alpha)$ is clearly inhabited. Furthermore, $f: X \rightarrow X$ defined as $f_{n}(i)=\min (i+1, n)$ and $f_{\omega}(i)=i+1$ is a non-expansive endomorphism. Yet it fails to have a fixpoint-i.e., a global element $\mathbf{1} \stackrel{c}{\rightarrow} X$ s.t. $f \circ c=c$.

Of course, we can do better in special cases.

Corollary 44. Whenever $X \in \mathscr{E}$ is s.t. any maximal subterminal subobject of $X$ is a global element (for example, $X$ is an injective object), there exists $\mathrm{fin} ! \stackrel{c}{\rightarrow} X$ s.t. $f \circ c=c$ for any non-expansive $f \in \mathscr{E}[X, X]$.

We could try to express unique existence in the internal logic using the standard abbreviation $\exists$ ! for "exists exactly one". However, as kindly pointed out by Thomas Streicher, this abbreviation works as intended in toposes of presheaves, but not necessarily in arbitrary ones.

But where is the place for a modality in all this? Say that $\odot: \Omega \rightarrow \Omega$ is a strong Löb operator if $\vDash_{\mathscr{E}} \forall p: \underline{\Omega}$. (@ $\left.p \rightsquigarrow p\right) \rightsquigarrow p$. Also, call a morphism $f \in \mathscr{E}[X, Z] \odot$-contractive if $\vDash_{\mathscr{E}} \forall x, y: \underline{X}$.@ $(x \approx y) \rightsquigarrow(\underline{f} x \approx \underline{f} y):$

\footnotetext{
${ }^{6}$ The corresponding theorem in EJP00 contained also an additional statement about density of the support of the fixed-point subobject, but this does not seem essential for us here.
} 
Corollary 45. Let $\odot: \Omega \rightarrow \Omega$ be a strong Löb operator, $f \in \mathscr{E}[X, X]$, and assume that $f$ is ○-contractive. Then $f$ is non-expansive and hence its subobject of fixed points is a maximal subterminal one.

Proof. We have that $\vDash_{\mathscr{E}} \forall p, q: \underline{\Omega}$. $(\odot p \rightsquigarrow(p \vee q)) \rightsquigarrow((q \rightsquigarrow p) \rightsquigarrow p)$. In fact, this is an equivalent form of (slöb) - cf. the proof of Theorem 2(iv) in [EJP00]. Now substitute $x \approx y$ for $p$ and $\underline{f} x \approx \underline{f} y$ for $q$ to get the result.

[EJP00 states the result only for a special case of contractiveness and a special subclass of toposes (introduced below) but this generalization is straightforward. As before, we can derive the conclusion about the existence of unique fixed points whenever every maximal subterminal object of $X$ happens to be a global element - e.g., whenever $X$ is injective.

Define $\odot \phi:=\forall t: \underline{\Omega} .(t \vee(t \rightsquigarrow \phi))$, i.e., an internalized coderivative. We have the following counterpart of Proposition 19 .

Proposition 46. In any elementary topos $\mathscr{E}$, we have $\vDash_{\mathscr{E}} \forall p: \underline{\Omega} . p \rightsquigarrow \bigodot p$ and $\vDash_{\mathscr{E}} \forall p, q$ : $\underline{\Omega} . \odot p \rightsquigarrow(q \vee(q \rightsquigarrow p))$.

A scattered topos is defined analogously to scattered locales or Heyting algebras in Section

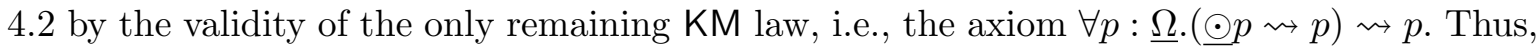
scattered toposes are those where $\odot$ is a strong Löb operator. This notion turns out to have several equivalent characterizations, see [EJP00]. Let us discuss in detail here another one for the special case of Set $^{\mathscr{R}}$ :

Theorem 47. Let $\mathscr{R}=(W, \unlhd)$ be a poset. Then $\operatorname{Set}^{\mathscr{R}}$ is scattered iff $(W, \unlhd, \triangleleft)$ satisfies any of the equivalent conditions in Corollary 26.

Proof. For a direct proof, it is useful to compute the semantic meaning of $\odot$. Define $\odot: \Omega \rightarrow \Omega$ as $[p: \underline{\Omega} \vdash \odot p: \underline{\Omega}]$. Recall also that in a topos of the form $\operatorname{Set}^{\mathscr{R}}$ for $\mathscr{\mathscr { R }}=(W, \unlhd), \Omega(w)$ is equal to $\left\{A \cap\{w\} \unlhd \uparrow \mid A \in \mathrm{Up}_{\unlhd}(W)\right\}$. A morphism $f: \Omega \rightarrow \Omega$ is a natural transformation: a family of mappings $\left\{f_{w}: \Omega(w) \rightarrow \Omega(w) \mid w \in W\right\}$ satisfying

$$
f_{z}(A \cap\{z\} \unlhd \uparrow)=f_{w}(A) \cap\{z\} \unlhd \uparrow \text { for any } A \in \Omega(w), z \unrhd w .
$$

Now let us note the following

Fact 48. For any topos of the form Set $^{\mathscr{R}}$ where $\mathscr{R}=(W, \unlhd)$, for any $w \in W$ and for any $A \in \Omega(w)$ (i.e., $A$ an upward closed subset of $\{w\} \unlhd \uparrow$ ),

$$
\odot_{w}(A)=\{z \unrhd w \mid\{z\} \triangleleft \uparrow \subseteq A\} .
$$

The reader may want to consult Section 4 and Table 3 for the notation used above; in particular, recall that $\{z\} \triangleleft \uparrow=\{z\} \unlhd \uparrow-\{z\}$. Note also that we can add an atomic clause to Corollary 39 in the preceding section:

Fact 49. $w, A \Vdash t$ (where $\Gamma=t: \underline{\Omega}$ and $A \in \Omega(w)$ ) iff $A=\{w\} \unlhd \uparrow$.

This fact, while rather basic, is worth an explicit proof, as it helps to put together several definitions and propositions above:

Proof of Fact 49 . $w, A \Vdash t$ is an abbreviation for $\operatorname{hom}_{\mathscr{R}}^{w}, \hat{A} \Vdash t$, while this in turn can be reformulated as $\hat{A}=$ true $\circ$ fin! hom $_{\mathscr{R}}^{w}$ (Fact 37 . In particular, $\hat{A}(w \unlhd w)=\{w\} \unlhd \uparrow$. But $\hat{A}(w \unlhd w)=A$. 
Fact 49 can be generalized with variable $t$ on the right hand side of the turnstile replaced with arbitrary $\phi(t)$. Somewhat informally speaking, $w, A \Vdash \phi(t)$ iff the value of $\phi(A)$ contains $\{w\} \unlhd \uparrow$ (think of $\phi$ here as a polynomial on the Heyting algebra of upward closed subsets of $\{w\} \unlhd \uparrow)$.

Putting all this together, we get that $w, A \Vdash(\odot p \rightsquigarrow p) \rightsquigarrow p$ iff for any $z \unrhd w$, it holds that $z, A \cap\{z\} \unlhd \uparrow \Vdash(\odot p \rightsquigarrow p)$ implies $\{z\} \unlhd \uparrow \subseteq A$. That is, $\left\{z^{\prime} \unrhd z \mid\left\{z^{\prime}\right\} \triangleleft \uparrow \subseteq A\right\} \subseteq A$ only if $\{z\} \unlhd \uparrow \subseteq A$ and in order for $\operatorname{Set}^{(W, \unlhd)}$ to be scattered this has to hold for any $w \in W$, any $z \unrhd w$ and any $A \in \Omega(w)$. But then the reasoning can be completed just like in the case of Corollary 26. This finishes the proof of Theorem 47 .

\subsection{Topos of Trees and Its Generalizations}

BMSS12 introduced the topos of trees (or forests) $\mathcal{S}$, i.e., the topos of presheaves on $(\omega, \unrhd)$, where $\unrhd$ is the converse of the usual order on $\omega$. Let us begin with the observation that an axiomatization of the $\mathrm{mHC}$-logic of the underlying frame of $\mathcal{S}$ can be obtained by means of techniques introduced in Section 4.3 .

Theorem 50. $\mathrm{KM} \oplus \mathrm{LC}$ is the propositional logic of $(\omega, \unrhd,>)$.

Proof (sketch). Just like in the first sentence of the proof of Corollary 32, we note that $\sigma^{\text {mix }}(\mathrm{KM} \oplus \mathrm{LC})$ is a just a notational variant of $\mathrm{GL}^{\mathrm{cl}} \cdot 3:=\mathrm{GL}^{\mathrm{cl}} \oplus$.3. This logic in turn can be shown (using standard modal techniques) to be the logic of natural numbers with reverse strict order taken as a classical Kripke frame. The result now follows from properties of $\sigma^{\text {mix. }}$. which are not quoted explicitly in Theorem 28, but can be extracted immediately from the original references, for example WZ97, Prop. 21].

Reasoning analogous to those in proofs of Corollary 39 and Theorem 47 yields then that $\mathrm{KM} \oplus \mathrm{LC}$ coincides with the set of $\mathcal{S}$-validities in the Mitchell-Bénabou language restricted to (topos-theoretical counterparts of) connectives in $\mathcal{L}_{\text {int }}$ together with $\odot$, the latter interpreted in in $\operatorname{Set}^{(\omega, \unrhd)}$ by $\odot$ as specified by Fact 48 above. Similar observations underly recent prooftheoretical investigations of this logic in [CG14].

Theorem 47 shows that the topos of trees is scattered. $\Omega$-endomorphism " $\triangleright$ " (this notation here would risk clashing with the one for a strict partial order and its converse) defined in BMSS12 is easily seen to coincide with $\odot$. The Internal Banach Fixpoint Theorem 2.9 of BMSS12 shows that $\odot$-contractive mappings on arbitrary inhabited objects in $\mathcal{S}$ do have (unique) fixpoints.

Now, Example 43 above shows that such a strong statement is not valid in arbitrary scattered toposes of presheaves, even quite similar to $\mathcal{S}$. The crucial Lemma 2.10 in [BMSS12] is not amenable to far-reaching generalizations.

However, BMSS12, Section 8] discusses a whole class of toposes together with a notion of a contractiveness guaranteeing fixpoint's existence. The class in question are sheaves on complete Heyting algebras with a well-founded basis DM04 rather than just presheaves on Noetherian partial orders - crucially, $\mathcal{S}$ can be also seen as such a sheaf topos-and the required notion of contractiveness is stronger than the one expressible in the internal logic.

Let us elaborate on the last point. As we saw, toposes allow an internal interpretation of modalities as morphisms $\Omega \rightarrow \Omega$. Actually, from the "propositions as predicates" perspective, any operation on subobjects of a given object is a "local" candidate for a modality. However, 
constructive or categorical logic is mostly about "propositions as types"; see, e.g., AMPR01; BPR01: PR11; BP00; PD01; PR11] for modal aspects. This perspective works even with mild assumptions about the underlying category. In particular, algebraic type theories require only finite products, whereas ccc's correspond to functional type theories [Cro93]: those whose type system is in fact that of Brouwerian semilattices of Remark 7 above. To see the details of this correspondence, just remove the rules for $\underline{\Omega}, \approx$ and all abbreviations using these from Tables 4 and 5 , then interpret conjunctions as products and implication as exponentation.

From this perspective, modalities correspond to endofunctors. In particular, ND systems for $\mathrm{PLL}^{\mathrm{i}}$ and $\mathrm{S}^{\mathrm{i}}$ are interpreted by, respectively, monads and comonads - see, e.g., references in Remark 17 and $\mathrm{SL}^{\mathrm{i}}$ yields a special subclass of pointed or applicative functors. MP08 More precisely, one obtains a variant of BMSS12, Definition 6.1]. Possible differences are: the modal assumption of normality forces only being monoidal wrt cartesian structure (cf. [BPR01; PR11]) rather than preservation of all finite limits as in the second clause of that definition; furthermore, the assumption of uniqueness in the first clause would rely on exact reduction and conversion rules of the proof system. A systematic study of such endofunctors in a cartesian setting - i.e., assuming only the presence conjunction among propositional connectives - has been undertaken by ML13] under the name of guarded fixpoint categories. Other possible names for such endofunctors include contraction, delay, (strong) Löb, $\mathrm{SL}^{\mathrm{i}}$ and $M G R T$, the last being an abbreviation of the original name in BMSS12.

One can relate these two views on modalities. Whenever $F: \mathscr{E} \rightarrow \mathscr{E}$ is monic-preserving and $\mathscr{E}$ has pullbacks, associate with a $F$-coalgebra $C \stackrel{\gamma}{\rightarrow} F C$ a modality $[F . \gamma]$ on subobjects $M \stackrel{m}{\longmapsto} C$ :

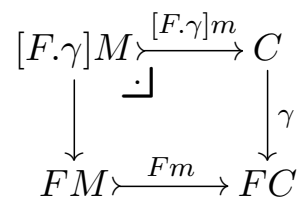

(see AMMS12 for the history of this diagram in papers on well-founded coalgebras). Furthermore, whenever $F$ is pointed (applicative), i.e., a $\mathrm{R}^{\mathrm{i}}$-endofunctor with $(F): 1 \rightarrow F$ being the point or unit of $F,(F)_{M}$ is a subcoalgebra of $(F)_{C}$ for any $M \stackrel{m}{\longmapsto} C$ and hence $m \leq\left[F .(F)_{C}\right] m$, i.e., the "local" translation of $(r)$ is universally valid.

Remark 51. [BMSS12, Theorem 6.8] allows to isolate sufficient conditions ensuring that the operator $\left[F .(F)_{C}\right]$ induced by a SLi-endofunctor $F: \mathscr{E} \rightarrow \mathscr{E}$ is a $\mathrm{SL}^{\mathrm{i}}$-modality: pullback-preservation of $F$ and $\mathscr{E}$ being a topos. While it is not mentioned in [BMSS12], one can find natural counterexamples when such conditions are dropped!

The operation on subobjects of $C \in \mathcal{S}$ induced by $\odot: \Omega \rightarrow \Omega$ from Fact 48 is defined in an alternative way in $\left[\right.$ BMSS12] : as $\left[\boldsymbol{\bullet}(\mathbf{(})_{C}\right]$ for a delay endofunctor $\bullet: \mathcal{S} \rightarrow \mathcal{S}$, whose action on objects is $(\bullet C)(0):=\mathbf{1}$ and $(\bullet C)(n+1):=(\bullet C)(n)$. In a sense, $\bullet$ can be called the Cantor-Bendixson endofunctor. Factoring through it is the desired "external" notion of contractivity ensuring fixpoint's existence. Both notions nicely complement each other:

... the external notion provides for a simple algebraic theory of fixed points for not only morphisms but also functors (see Section 2.6), whereas the internal notion is 
useful when working in the internal logic. BMSS12

Could [EJP00] have had more impact if the authors had a) employed the external perspective on modalities in addition to the internal one and b) had the hindsight of [DM04]? This is rather too counterfactual a question to consider. Note also that what matters from the point of view of BMSS12] - and Theoretical CS in general - is the use made of these external and internal Löb modalities. BMSS12, Section 3] constructs a model of a programming language with higher-order store and recursive types entirely inside the internal logic of $\mathcal{S}$. BMSS12, Section 4] provides semantic foundation for dependent type theories extended with a SL' modality and guarded recursive types; this can be regarded as an extension of fixpoint results along the lines of Section 3 above to predicate and higher-order constructive logics. [BMSS12, Section 5] shows that a class of (ultra-)metric spaces commonly used in modelling corecursion on streams is equivalent to a subcategory of $\mathcal{S}$.

Clearly, this is a large area rather overlooked by researchers on (intuitionistic) modal logic side. There is no space here to discuss my own work in progress, e.g., on the Curry-Howard interpretation of $\mathrm{mHC}$, but let us conclude with a question from participants of ToLo III: is there a natural subclass of internal modalities in toposes (endomorphisms $\Omega \rightarrow \Omega$ ) inducing external modalities (endofunctors) in a generic way?

Remark 52. Earlier incarnations of this paper included also a question by Lars Birkedal: what are additional logical principles which would allow a scattered topos to model not only guarded (co-)recursion, but also, e.g., countable nondeterminism? However, a recent work [BBM14] addressed the issue: expressing the additional property needed for stating the adequacy of the logical relation requires more than logical connectives introduced so far. It can be only stated in presence of an additional modality right adjoint to double negation.

Acknowledgements Special thanks are due to: the anonymous referee for careful reading and many helpful comments; Guram Bezhanishvili, for an invitation to write this chapter, but also his tolerance, understanding and patience during the whole process; Alexander Kurz for his support, interest and numerous discussions; Mamuka Jibladze and the late Dito Pataraia, particularly for all the discussions we had in Kutaisi. Apart from this, the list of people who provided feedback and/or suggestions include (in no particular order) Lars Birkedal, Rasmus Møgelberg, Stefan Milius, Thomas Streicher, Sam Staton, Alex Simpson, Gordon Plotkin, Johan van Benthem, Ramon Jansana, Achim Jung, Andrew Pitts, Conor McBride, Nick Benton, Guilhem Jaber, Dirk Pattinson, Drew Moshier, Nick Bezhanishvili, Alexei Muravitsky, Fredrik Nordvall Forsberg, Ben Kavanagh, Oliver Fasching, participants of Domains X, TYPES 2011, MGS 2012 and seminars at the University of Leicester. For the extended version, I would also like to acknowledge Ranald Clouston, Rajeev Goré and Aleš Bizjak. During early stages of the write-up process, I was supported by the EPSRC grant EP/G041296/1.

\section{References}

[Aba07] M. Abadi. "Access Control in a Core Calculus of Dependency". In: Electr. Notes Theor. Comput. Sci. 172 (2007), pp. 5-31. 
[Acz01] P. Aczel. "The Russell-Prawitz modality". In: Mathematical Structures in Computer Science 11.4 (2001), pp. 541-554.

[AMMS12] J. Adámek, S. Milius, L. S. Moss, and L. Sousa. "Well-pointed Coalgebras (Extended Abstract)". In: Proc. Fifteenth International Conference on Foundations of Software Science and Computation Structures (FoSSaCS'12). Ed. by L. Birkedal. Vol. 7213. Lecture Notes Comput. Sci. (ARCoSS). 2012, pp. 89-103.

[AF09] L. Alberucci and A. Facchini. "On Modal $\mu$-Calculus and Gödel-Löb Logic". In: Studia Logica 91.2 (2009), pp. 145-169.

[AMPR01] N. Alechina, M. Mendler, V. de Paiva, and E. Ritter. "Categorical and Kripke Semantics for Constructive S4 Modal Logic". In: Computer Science Logic (CSL). Ed. by L. Fribourg. Vol. 2142. Lecture Notes in Computer Science. EACSL. Springer, 2001, pp. 292-307.

[Ame96] M. Amerbauer. "Cut-Free Tableau Calculi for Some Propositional Normal Modal Logics". In: Studia Logica: An International Journal for Symbolic Logic 57.2/3 (1996), pp. 359372. URL: http://www.jstor .org/stable/20015881.

[AMRV07] A. W. Appel, P.-A. Melliès, C. D. Richards, and J. Vouillon. "A very modal model of a modern, major, general type system". In: Principles of Programming Languages (POPL). Ed. by M. Hofmann and M. Felleisen. ACM SIGPLAN-SIGACT. 2007, pp. 109-122.

[AM13] R. Atkey and C. McBride. "Productive coprogramming with guarded recursion". In: International Conference on Functional Programming, (ICFP). Ed. by G. Morrisett and T. Uustalu. ACM SIGPLAN. 2013, pp. 197-208.

[BPR01] G. Bellin, V. de Paiva, and E. Ritter. "Extended Curry-Howard Correspondence for a Basic Constructive Modal Logic". In: Proceedings of Methods for Modalities. 2001. URL: http://profs.sci.univr.it/\%5Ctextasciitilde $\% 20$ bellin/m4m.ps.

[Ben06] J. van Benthem. "Modal Frame Correspondences and Fixed-Points". In: Studia Logica 83.1-3 (2006), pp. 133-155.

[Ben91] J. van Benthem. "Reflections on epistemic logic". In: Logique et Analyse (1991), pp. 5-14.

[BT09] N. Benton and N. Tabareau. "Compiling functional types to relational specifications for low level imperative code". In: Types in Languages Design and Implementation (TLDI). Ed. by A. Kennedy and A. Ahmed. ACM SIGPLAN. 2009, pp. 3-14.

[BBP98] P. N. Benton, G. M. Bierman, and V. de Paiva. "Computational Types from a Logical Perspective". In: J. Funct. Program. 8.2 (1998), pp. 177-193.

[Bez14] G. Bezhanishvili, ed. Vol. 4. Outstanding Contributions to Logic. Springer, 2014.

[BJ13] G. Bezhanishvili and R. Jansana. "Esakia Style Duality for Implicative Semilattices". In: Applied Categorical Structures 21 (2013). 10.1007/s10485-011-9265-0, pp. 181-208. URL: http://dx.doi.org/10.1007/s10485-011-9265-0.

[BP00] G. M. Bierman and V. C. V. d. Paiva. "On an Intuitionistic Modal Logic". In: Studia Logica: An International Journal for Symbolic Logic 65.3 (2000), pp. 383-416. URL: http: //www.jstor.org/stable/20016199.

[Bir12] R. C. E. Biraben. "On Some Extensions of Intuitionistic Logic". In: Bulletin of the Section of Logic 41.1-2 (2012), pp. 17-22.

[BM13] L. Birkedal and R. E. Møgelberg. "Intensional Type Theory with Guarded Recursive Types qua Fixed Points on Universes". In: Logic in Computer Science (LICS). ACM/IEEE. 2013, pp. 213-222.

[BMSS12] L. Birkedal, R. E. Møgelberg, J. Schwinghammer, and K. Støvring. "First Steps in Synthetic Guarded Domain Theory: Step-Indexing in the Topos of Trees". In: Logical Methods in Computer Science 8 (4 2012), pp. 1-45.

[BBM14] A. Bizjak, L. Birkedal, and M. Miculan. "A Model of Countable Nondeterminism in Guarded Type Theory". In: Rewriting and Typed Lambda Calculi. Ed. by G. Dowek. Vol. 8560. Lecture Notes in Computer Science. Springer International Publishing, 2014, pp. 108-123. URL: http://dx.doi.org/10.1007/978-3-319-08918-8_8.

[BdV01] P. Blackburn, M. de Rijke, and Y. Venema. Modal Logic. Cambridge University Press, 2001.

[BP89] W. J. Blok and D. Pigozzi. Algebraizable logics. Vol. 77 (396). Memoirs AMS. AMS, 1989. 
[Boo93] G. Boolos. The Logic of Provability. Cambridge University Press, 1993.

[BS91] G. Boolos and G. Sambin. "Provability: the emergence of a mathematical modality". In: Studia Logica 50 (1 1991). 10.1007/BF00370383, pp. 1-23. URL: http://dx.doi.org/ 10.1007/BF00370383.

[BD84] M. Božić and K. Došen. "Models for Normal Intuitionistic Modal Logics". In: Studia Logica: An International Journal for Symbolic Logic 43.3 (1984), pp. 217-245. URL: http: //www.jstor.org/stable/20015164

[BS81] S. Burris and H. Sankappanavar. A Course in Universal Algebra. Graduate Texts in Mathematics. Springer-Verlag, New York, 1981.

[CFL10] B. ten Cate, G. Fontaine, and T. Litak. "Some modal aspects of XPath". In: Journal of Applied Non-Classical Logics 20.3 (2010), pp. 139-171.

[CZ97] A. Chagrov and M. Zakharyaschev. Modal Logic. Oxford Logic Guides 35. Clarendon Press, 1997.

[CG14] R. Clouston and R. Goré. "Sequent Calculus in the Topos of Trees". work in progress. 2014.

[Cro93] R. L. Crole. Categories for Types. Cambridge Mathematical Textbooks. xvii +335 pages, ISBN 0521450926HB, 0521457017PB. Cambridge University Press, 1993.

[Cur52] H. B. Curry. "The Elimination Theorem When Modality is Present". In: The Journal of Symbolic Logic 17.4 (1952), pp. 249-265. URL: http://www.jstor.org/stable/2266613.

[DM04] P. Di Gianantonio and M. Miculan. "Unifying Recursive and Co-recursive Definitions in Sheaf Categories". In: Foundations of Software Science and Computation Structures. Ed. by I. Walukiewicz. Vol. 2987. Lecture Notes in Computer Science. 10.1007/978-3540-24727-2_11. Springer Berlin / Heidelberg, 2004, pp. 136-150. URL: http://dx.doi . org/10.1007/978-3-540-24727-2\%5C_11.

[Doš85] K. Došen. "Models for Stronger Normal Intuitionistic Modal Logics". In: Studia Logica 44.1 (1985), pp. 39-70. URL: http://www.jstor.org/stable/20015197.

[Esa98] L. Esakia. "Quantification in Intuitionistic Logic with Provability Smack". In: Bulletin of the Section of Logic 27 (1998), pp. 26-28.

[Esa06] L. Esakia. "The modalized Heyting calculus: a conservative modal extension of the Intuitionistic Logic". In: Journal of Applied Non-Classical Logics 16.3-4 (2006), pp. 349366.

[EJP00] L. Esakia, M. Jibladze, and D. Pataraia. "Scattered Toposes". In: Ann. Pure Appl. Logic 103.1-3 (2000), pp. 97-107.

[FM97] M. Fairtlough and M. Mendler. "Propositional Lax Logic". In: Inf. Comput. 137.1 (1997), pp. 1-33.

[Fin85] K. Fine. "Logics Containing K4. Part II". In: The Journal of Symbolic Logic 50.3 (1985), pp. 619-651. URL: http://www.jstor.org/stable/2274318.

[Fon06] J. M. Font. "Beyond Rasiowa's Algebraic Approach to Non-Classical Logics". In: Studia Logica 82.2 (2006), pp. 179-209. URL: http://www.jstor.org/stable/20016776.

[FJP03] J. M. Font, R. Jansana, and D. Pigozzi. "A Survey of Abstract Algebraic Logic". In: Studia Logica 74.1-2 (2003), pp. 13-97.

[FJP09] J. M. Font, R. Jansana, and D. Pigozzi. "Update to "A Survey of Abstract Algebraic Logic"”. In: Studia Logica 91.1 (2009), pp. 125-130.

[GA08] D. Garg and M. Abadi. "A Modal Deconstruction of Access Control Logics". In: Foundations of Software Science and Computational Structures (FOSSACS). Ed. by R. M. Amadio. Vol. 4962. Lecture Notes in Computer Science. Springer, 2008, pp. 216-230.

[GP06] D. Garg and F. Pfenning. "Non-Interference in Constructive Authorization Logic". In: 19th IEEE Computer Security Foundations Workshop, (CSFW-19). IEEE Computer Society. 2006, pp. 283-296.

[Ghi89] S. Ghilardi. "Presheaf semantics and independence results for some non-classical firstorder logics". In: Archive for Mathematical Logic 29 (2 1989). 10.1007/BF01620621, pp. 125-136. URL: http://dx.doi.org/10.1007/BF01620621. 
[Gol06] R. Goldblatt. Topoi: the categorial analysis of logic. Dover Books on Mathematics. Dover Publications, 2006.

[Gol03] R. Goldblatt. "Mathematical modal logic: A view of its evolution". In: Journal of Applied Logic 1.5-6 (2003), pp. 309-392. URL: http : / / www . sciencedirect . com / science / article/pii/S1570868303000089.

[Gol10] R. I. Goldblatt. "Cover semantics for quantified lax logic". In: Journal of Logic and Computation (2010), pp. 1035-1063.

[Gol81] R. I. Goldblatt. "Grothendieck Topology as Geometric Modality". In: Mathematical Logic Quarterly 27.31-35 (1981), pp. 495-529. URL: http://dx.doi.org/10 .1002/malq. 19810273104 .

[Hor62] A. Horn. "The Separation Theorem of Intuitionist Propositional Calculus". In: The Journal of Symbolic Logic 27.4 (1962), pp. 391-399. URL: http://www.jstor.org/stable/ 2964545 .

[Iem01] R. Iemhoff. "Provability logic and admissible rules". PhD thesis. University of Amsterdam, 2001.

[JTS12] G. Jaber, N. Tabareau, and M. Sozeau. "Extending Type Theory with Forcing". In: Logic in Computer Science (LICS). IEEE. 2012, pp. 395-404.

[Joh02] P. Johnstone. Sketches of an Elephant: A Topos Theory Compendium. Oxford Logic Guides. Clarendon Press, 2002.

[JV95] D. de Jongh and A. Visser. Embeddings of Heyting Algebras. ILLC research report and technical notes series. Institute for Logic, Language and Computation, University of Amsterdam, 1995. URL: http://books.google.de/books?id=2AMjSAAACAAJ.

[Köh81] P. Köhler. "Brouwerian Semilattices". In: Transactions of the American Mathematical Society 268.1 (1981), pp. 103-126. URL: http://www.jstor.org/stable/1998339.

[KB11a] N. R. Krishnaswami and N. Benton. "A semantic model for graphical user interfaces". In: International Conference on Functional Programming, (ICFP). Ed. by M. M. T. Chakravarty, Z. Hu, and O. Danvy. ACM SIGPLAN. ACM, 2011, pp. 45-57.

[KB11b] N. R. Krishnaswami and N. Benton. "Ultrametric Semantics of Reactive Programs". In: Logic in Computer Science (LICS). IEEE. 2011, pp. 257-266.

[Kuz79] A. V. Kuznetsov. "On algebras of open sets". In: The fourth Tiraspol symposium on general topology and its applications. Abstracts, Kishinev (Russian). 1979, pp. 72-75.

[KM86] A. V. Kuznetsov and A. Y. Muravitsky. "On Superintuitionistic Logics as Fragments of Proof Logic Extensions". In: Studia Logica: An International Journal for Symbolic Logic 45.1 (1986), pp. 77-99. URL: http://www.jstor.org/stable/20015249.

[LS86] J. Lambek and P. J. Scott. Introduction to Higher Order Categorical Logic. Cambridge studies in advanced mathematics 7. Cambridge University Press, 1986.

[Lit14] T. Litak. "Constructive modalities with provability smack". In: Leo Esakia on Duality in Modal and Intuitionistic Logics. Ed. by G. Bezhanishvili. Vol. 4. Outstanding Contributions to Logic. Springer, 2014, pp. 179-208.

[Lit07] T. Litak. "The Non-reflexive Counterpart of Grz". In: Bulletin of the Section of Logic 36 (2007), pp. 195-208.

[MM92] S. MacLane and I. Moerdijk. Sheaves in Geometry and Logic: A First Introduction to Topos Theory. Corrected. Springer, 1992.

[MP08] C. McBride and R. Paterson. "Applicative programming with effects". In: J. Funct. Program. 18.1 (2008), pp. 1-13.

[McL90] C. McLarty. "The Uses and Abuses of the History of Topos Theory". In: British Journal for the Philosophy of Science 41.3 (1990), pp. 351-375.

[ML13] S. Milius and T. Litak. "Guard Your Daggers and Traces: On The Equational Properties of Guarded (Co-)recursion". In: Fixed Points in Computer Science (FICS). Ed. by D. Baelde and A. Carayol. Vol. 126. EPTCS. Soon to become obsolete, journal version submitted. 2013, pp. 72-86. URL: http://dx.doi.org/10.4204/EPTCS.126.

[Mil10] P. Milne. "Subformula and separation properties in natural deduction via small kripke models". In: The Review of Symbolic Logic 3.02 (2010), pp. 175-227. eprint: http: // 
journals . cambridge .org/article_S175502030999030X. URL: http://dx.doi.org/ 10.1017/S175502030999030X.

[Mos07] L. Moss. "Finite models constructed from canonical formulas". In: Journal of Philosophical Logic 36 (6 2007). 10.1007/s10992-007-9052-4, pp. 605-640. URL: http://dx.doi. org/10.1007/s10992-007-9052-4.

[Mur81] A. Y. Muravitsky. "Finite approximability of the calculus $\mathrm{I}^{\Delta}$ and the nonmodelability of some of its extensions". In: Mat. Zametki 29.6 (1981), pp. 907-916, 957.

[Mur14] A. Y. Muravitsky. "Logic KM: A Biography". In: Leo Esakia on Duality in Modal and Intuitionistic Logics. Ed. by G. Bezhanishvili. Vol. 4. Outstanding Contributions to Logic. Springer, 2014, pp. 147-178.

[NI97] K. Nagaoka and E. Isoda. "Incompleteness Results in Kripke Bundle Semantics". In: Mathematical Logic Quarterly 43.4 (1997), pp. 485-498. URL: http://dx.doi.org/10. 1002/malq.19970430405.

[Nak00] H. Nakano. "A Modality for Recursion". In: Logic in Computer Science (LiCS). IEEE. 2000, pp. 255-266.

[Nak01] H. Nakano. "Fixed-Point Logic with the Approximation Modality and Its Kripke Completeness". In: Theoretical Aspects of Computer Software (TACS). Ed. by N. Kobayashi and B. C. Pierce. Vol. 2215. Lecture Notes in Computer Science. Springer, 2001, pp. 165182.

[PR11] V. de Paiva and E. Ritter. "Basic Constructive Modality". In: Logic Without FrontiersFestschrift for Walter Alexandre Carnielli on the occasion of his 60th birthday. Ed. by J.-Y. Beziau and M. E. Coniglio. College Publications, 2011, pp. 411-428.

[PD01] F. Pfenning and R. Davies. "A judgmental reconstruction of modal logic". In: Mathematical Structures in Computer Science 11.4 (2001), pp. 511-540.

[PS86] G. D. Plotkin and C. Stirling. "A Framework for Intuitionistic Modal Logics". In: Theoretical Aspects of Reasoning about Knowledge (TARK). Ed. by J. Y. Halpern. Morgan Kaufmann, 1986, pp. 399-406.

[Pra65] D. Prawitz. "Natural deduction: a proof-theoretical study". PhD thesis. Almqvist \& Wiksell, 1965.

[Ras74] H. Rasiowa. An Algebraic Approach to Non-classical Logics. North Holland, 1974.

[Sam76] G. Sambin. "An Effective Fixed-Point Theorem in Intuitionistic Diagonalizable Algebras (The Algebraization of the Theories Which Express Theor, IX)". In: Studia Logica 35.4 (1976), pp. 345-361. URL: http://www.jstor.org/stable/20014825.

[Sim82] H. Simmons. "An algebraic version of Cantor-Bendixson analysis". In: Categorical Aspects of Topology and Analysis. Ed. by B. Banaschewski. Vol. 915. Lecture Notes in Mathematics. 10.1007/BFb0092888. Springer Berlin / Heidelberg, 1982, pp. 310-323. URL: http://dx.doi.org/10.1007/BFb0092888.

[Sim14] H. Simmons. "Cantor-Bendixson properties of the assembly of a frame". In: Leo Esakia on Duality in Modal and Intuitionistic Logics. Ed. by G. Bezhanishvili. Vol. 4. Outstanding Contributions to Logic. Springer, 2014.

[Sim94] A. K. Simpson. "The Proof Theory and Semantics of Intuitionistic Modal Logic". PhD thesis. University of Edinburgh, 1994. URL: http://homepages .inf .ed.ac.uk/als/ Research/thesis.ps.gz.

[Skv12] D. Skvortsov. "Kripke Sheaf Completeness of some Superintuitionistic Predicate Logics with a Weakened Constant Domains Principle". In: Studia Logica 100 (1-2 2012), pp. 361383. URL: http://dx.doi.org/10.1007/s11225-012-9382-2.

[Smo79] C. Smorynski. "Calculating Self-Referential Statements, I: Explicit Calculations". In: Studia Logica 38.1 (1979), pp. 17-36. URL: http://www.jstor.org/stable/20014926.

[Sol76] R. M. Solovay. "Provability Interpretations of Modal Logic". In: Israel Journal of Mathematics 25 (1976), pp. 287-304.

[Sot84] V. Sotirov. "Modal theories with intuitionistic logic". In: Mathematical Logic, Proc. Conf. Math. Logic Dedicated to the Memory of A. A. Markov (1903-1979), Sofia, September 22 - 23, 1980. 1984, pp. 139-171. 
[Urs79] A. Ursini. "A Modal Calculus Analogous to K4W, Based on Intuitionistic Propositional Logic, I". In: Studia Logica 38.3 (1979), pp. 297-311. URL: http://www . jstor .org/ stable/20014950.

[Vak81] D. Vakarelov. "Intuitionistic Modal Logics Incompatible with the Law of the Excluded Middle". In: Studia Logica 40.2 (1981).

[Vis05] A. Visser. "Löb's Logic Meets the $\mu$-calculus". In: Processes, Terms and Cycles: Steps on the Road to Infinity, Essays Dedicated to Jan Willem Klop, on the Occasion of His 60th Birthday. Ed. by A. Middeldorp, V. van Oostrom, F. van Raamsdonk, and R. C. de Vrijer. Vol. 3838. Lecture Notes in Computer Science. Springer, 2005, pp. 14-25.

[Wol97] F. Wolter. "Superintuitionistic Companions of Classical Modal Logics". In: Studia Logica 58.2 (1997), pp. 229-259.

[WZ98] F. Wolter and M. Zakharyaschev. "Intuitionistic Modal Logics as fragments of Classical Modal Logics". In: Logic at Work, Essays in honour of Helena Rasiowa. Ed. by E. Orlowska. Springer-Verlag, 1998, pp. 168-186.

[WZ14] F. Wolter and M. Zakharyaschev. "On the Blok-Esakia Theorem". In: Leo Esakia on Duality in Modal and Intuitionistic Logics. Ed. by G. Bezhanishvili. Vol. 4. Outstanding Contributions to Logic. Springer, 2014, pp. 91-110.

[WZ97] F. Wolter and M. Zakharyaschev. "On the relation between intuitionistic and classical modal logics". In: Algebra and Logic 36 (1997), pp. 121-125. 